\title{
A Versatile Open-source Photoreactor Architecture for Photocatalysis Across the Visible Spectrum
}

Philip P. Lampkin, Blaise J. Thompson and Samuel H. Gellman*

University of Wisconsin-Madison, Madison, Wisconsin, 53706

Email: gellman@chem.wisc.edu

Supporting Information 


\section{Table of Contents}

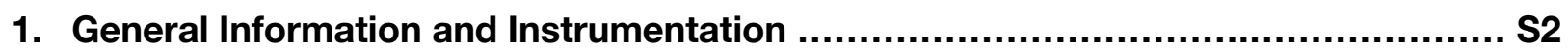

2. Wisconsin Photoreactor Platform Project Information ....................................... S3

2.1 Project Repository

2.2 Fabrication and Operation Information

2.3 Reproduction and Distribution Guidelines

2.4 Safety Notice

3. Photoreaction Setups

3.1 WPP Apparatus Setups

3.2 Comparison Setups

4. Photon Source Characterization

4.1 WPP Photon Source Emission Profiles

4.2 Comparison Setups Emission Profiles

5. Experimental Procedures

5.1 Benchmark 1 - C-N Cross-coupling of 4-Bromobenzotrifluoroide and Morpholine via Photoexcitation of Ni-amine Complexes

5.2 Benchmark 2 - Vitamin-catalyzed Synthesis of 3,4-Benzocoumarin

5.3 Benchmark 3 - Os-photocatalyzed Trifluoromethylation of N-Methyl-2-pyridone

5.4 Benchmark 4 - Trifluoromethylation of 1,3,5-Trimethoxybenzene

5.5 Benchmark 5 - Ru-photocatalyzed Trifluoromethylation of 2-Acetyl-N-boc-pyrrole

5.6 Benchmark 6 - C-N Cross-coupling of 4-Bromobenzotrifluoroide and Morpholine via Metallophotoredox catalysis

5.7 Reaction Monitoring by NMR

6. Additional Experiments and Information

6.1 Position Equivalency Experiment

6.2 Scale-up Experiment

6.3 Cooling Efficiency With and Without Light Shield Module

6.4 Comparison to MacMillan Photoreactor

7. Product Spectral Data .

8. References

9. Acknowledgments 


\section{General Information and Instrumentation}

Solvents and reagents were purchased from Sigma-Aldrich, TCl, Chem Impex, Strem Chemicals or Alfa-Aesar and used as received without further purification. Solvents used were ACS grade. All reactions were carried out under a nitrogen atmosphere using oven-dried glassware unless otherwise noted. Thin-layer chromatography was carried out using SiliCycle SiliaPlate $250 \mu \mathrm{m}$ extra hard layer $60 \AA$ plates. Spots were visualized with UV. Flash column chromatography was conducted using Silicycle SiliaFlash P60, 40-63 $\mu \mathrm{m} 60 \AA$ silica. High performance liquid chromatography was conducted using a Waters HPLC system (2707 autosampler, 2998 Photodiode Array Detector, 2545 Binary Gradient Module and Fraction Collector III) equipped with a Waters XSelect CSH C18 OBD Prep column (130 ̊, $5 \mu \mathrm{m}, 19 \mathrm{~mm}$ X $250 \mathrm{~mm})$.

All ${ }^{1} \mathrm{H},{ }^{13} \mathrm{C}$ and ${ }^{19} \mathrm{~F}$ spectra were taken on Bruker Avance 400 or Bruker Avance 600 NMR spectrometers at ambient temperatures. Deuterated solvents were purchased from SigmaAldrich. Chemical shifts $(\delta)$ are reported in parts per million (ppm). All ${ }^{1} \mathrm{H}$ and ${ }^{13} \mathrm{C}$ NMR spectra are internally referenced to tetramethylsilane $\left(\delta\left({ }^{1} \mathrm{H}\right)=0.00 \mathrm{ppm}, \delta\left({ }^{13} \mathrm{C}\right)=0.00 \mathrm{ppm}\right)$. Data for ${ }^{1} \mathrm{H}$ and ${ }^{13} \mathrm{C}$ NMR signal multiplicity are reported as $\mathrm{s}$ (singlet), $\mathrm{d}$ (doublet), $\mathrm{t}$ (triplet), $\mathrm{q}$ (quartet) or $\mathrm{m}$ (multiplet) and coupling constants $(\mathcal{J})$ are reported in $\mathrm{Hz}$. Signal data for ${ }^{19} \mathrm{~F}$ NMR spectra are reported only in terms of chemical shift.

Photon source emission spectra were obtained using an Ocean Insight USB2000 spectrometer in a dark room.

Wisconsin Photoreactor Platform enclosure components were designed using Autodesk Fusion 360. Electronic circuit boards were designed using KiCAD.

3D-printing of Wisconsin Photoreactor Platform enclosure components was accomplished using Creality Ender 3, Ultimaker 3 Extended or Stratasys uPrint SE Plus 3D printers. PrusaSlicer was used as the slicer program. Parts fabricated using the Creality Ender 3 or Ultimaker 3 Extended printers were printed with $10 \%$ infill using the " $0.24 \mathrm{~mm}$ DRAFT" and "brim enabled" settings in PrusaSlicer. The "supports on the build plate only" setting in PrusaSlicer was enabled when printing the Wisconsin Photoreactor Platform enclosure base. PLA filament from Hatchbox, Overture, ERYONE or SUNLU was used when printing with the Creality Ender 3 or Ultimaker 3 Extended printers. Parts fabricated with the Stratasys uPrint SE Plus 3D printer were printed using Stratasys ABS-P430 XL filament. 


\section{Wisconsin Photoreactor Platform Project Information}

\subsection{Project Repository}

All Wisconsin Photoreactor Platform (WPP) designs, schematics and other project source files as of June 2021 are supplied in the compressed project repository provided alongside this document.

The most up to date Wisconsin Photoreactor Platform project repository is available on GitHub (https://github.com/uw-madison-chem-shops/wisconsin-photoreactor).

\subsection{Fabrication and Operation Information}

Fabrication and operation instructions are supplied in the fabrication and operation guide provided alongside this document. A bill of materials for each component is provided in the README file located in each component's subdirectory in the project repository.

The most up to date fabrication and operation information is available on the Wisconsin Photoreactor Platform project repository on GitHub: https://github.com/uw-madison-chemshops/wisconsin-photoreactor.

\subsection{Reproduction and Distribution Guidelines}

The Wisconsin Photoreactor Platform is an open-source hardware project licensed under the "CERN Open Hardware License Version 2 - Permissive." This license provides a framework which supports the freedom to use, reproduce, modify, share and distribute the Wisconsin Photoreactor Platform.

Please see the LICENSE file in the WPP project repository for the complete license and guidelines.

\subsection{Safety Notice}

WPP apparatuses utilize high-intensity light emitting diodes (LED) that can cause eye damage if proper safety precautions are not observed.

A light-blocking shield should be utilized whenever operating a WPP apparatus. Care must be taken to ensure the light-blocking shield employed adequately blocks light emitted by a WPP photon source from directly reaching a user. A 3D-printable light blocking cover module compatible with WPP devices is provided in the project repository. Use of light-filtering safety glasses alongside a light-blocking shield can provide additional protection. See the fabrication and operation guide provided alongside this document for additional safety information. 


\section{Photoreaction Setups}

\subsection{WPP Apparatus Setups}
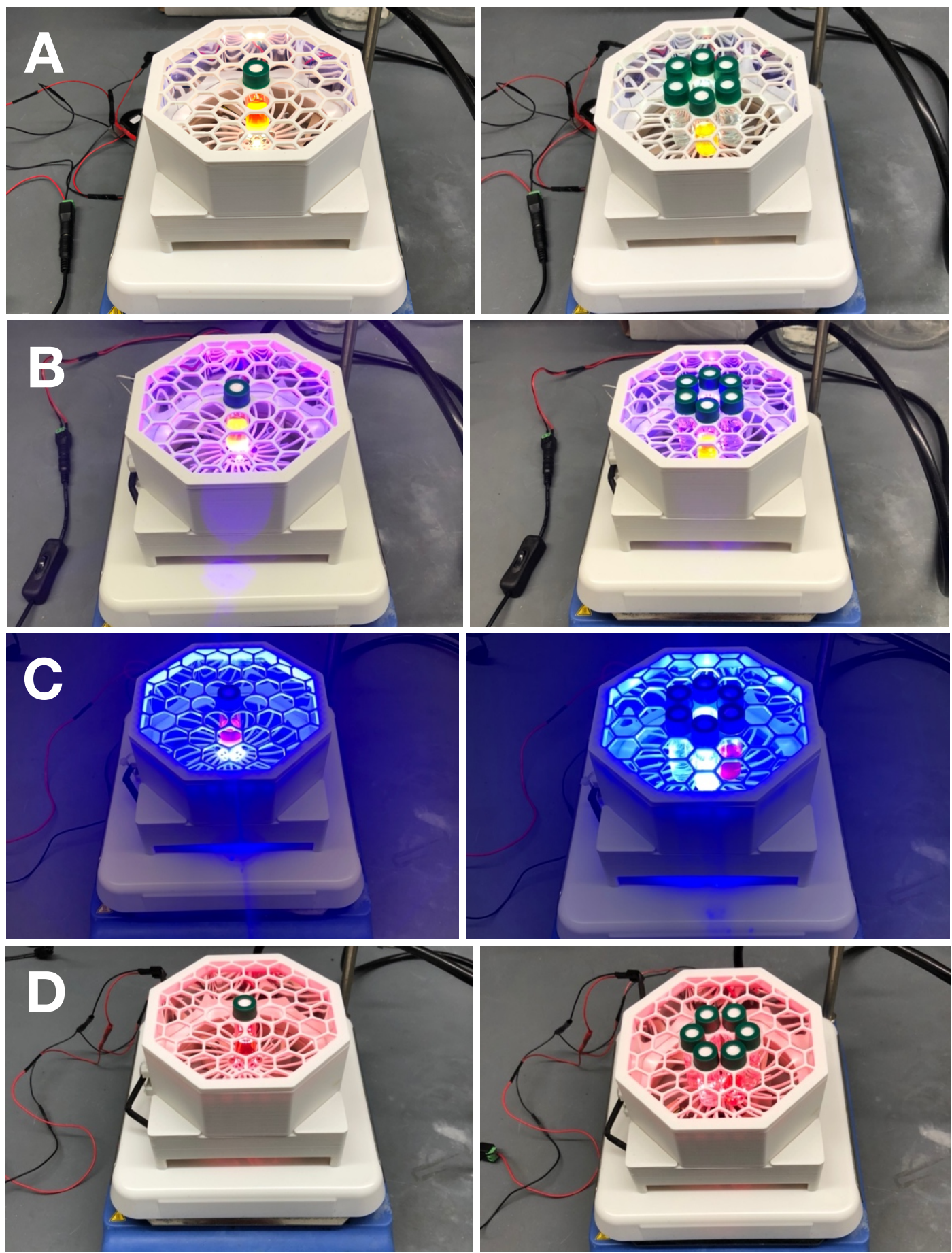

Figure S1: (A) A $365 \mathrm{~nm}$ WPP fitted with a 4-mL reaction module in the single and multiple reaction configurations. (B) $395 \mathrm{~nm}$ WPP device. (C) $450 \mathrm{~nm}$ WPP device. (D) $730 \mathrm{~nm}$ WPP device. 
Benchmarking experiments procedure: A WPP base fitted with a $365 \mathrm{~nm}, 395 \mathrm{~nm}, 450 \mathrm{~nm}$ or $730 \mathrm{~nm}$ LED star was connected to a simple LED driver circuit containing a $1000 \mathrm{~mA}$ LUXdrive PowerPuck LED driver (manufacturer part number 2008B-1000). The base was then fitted with a 4-mL reaction module, and the assembled apparatus was placed onto a standard laboratory stir plate behind a light blocking shield. For reactions conducted using the single reaction configuration, a single $4 \mathrm{~mL}$ reaction vial was inserted into the center of the module and held 7 $\mathrm{mm}$ above the photon source. For reactions conducted using the multiple reaction configuration, five $4 \mathrm{~mL}$ vials containing $2 \mathrm{~mL}$ of water and the $4 \mathrm{~mL}$ reaction vial were inserted around the module center and held $7 \mathrm{~mm}$ above the photon source. The apparatus was turned on by plugging the WPP device into a 12 Volt 2 Amp power supply. The reaction was left to stir for the desired time.

For the "WPP - $450 \mathrm{~nm}$ - single reaction" trial of benchmark reaction 6 , an analog driver board was used in place of the simple LED driver circuit, and the apparatus was placed on a stir plate situated in a $4{ }^{\circ} \mathrm{C}$ refrigerator. LED intensity was adjusted to $90 \%$ (measured test point voltage was $2.25 \mathrm{~V}$ ) using the potentiometer mounted to the analog board.

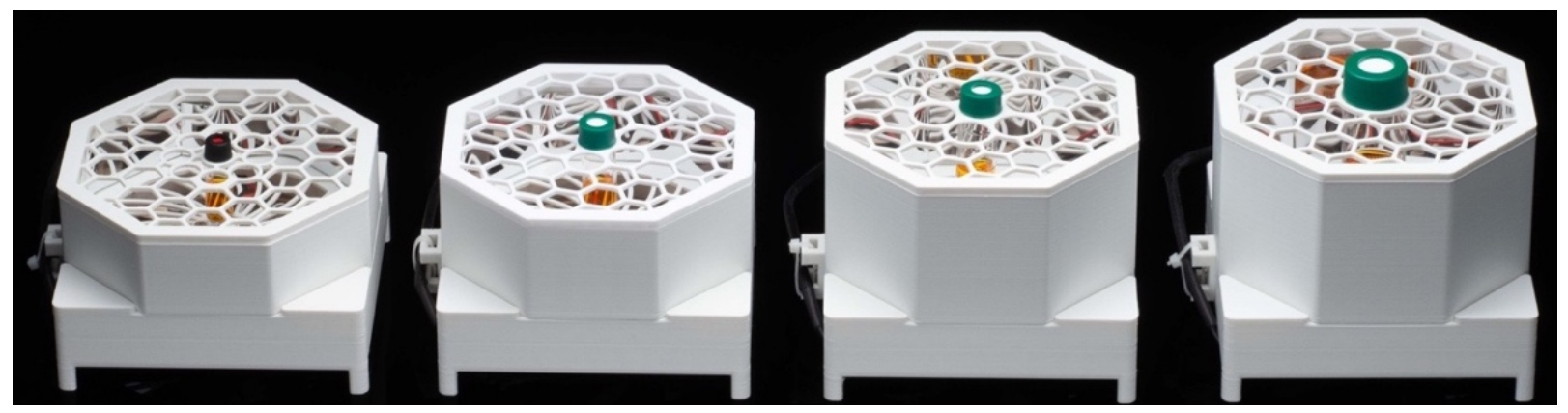

Figure S2: WPP devices fitted with 2-, 4-, 8- and 24-mL modules

Scale-up experiment procedure: A WPP base fitted with a $730 \mathrm{~nm}$ LED star was connected to a simple LED driver circuit containing a $1000 \mathrm{~mA}$ LUXdrive PowerPuck LED driver (manufacturer part number 2008B-1000). The base was fitted with a 2-, 4-, 8- or 24-mL reaction module, and the assembled apparatus was placed onto a standard laboratory stir plate. A single reaction vial was then inserted into the center of the module and held $7 \mathrm{~mm}$ above the photon source. The apparatus was turned on by plugging the WPP device into a standard 12 Volt 2 Amp power supply and the reaction mixture was left to stir for the desired time.

Position equivalency experiments procedure: A WPP base fitted with a $450 \mathrm{~nm}$ LED star was connected to a simple LED driver circuit containing a 1000 mA LUXdrive PowerPuck LED driver (manufacturer part number 2008B-1000). The base was then fitted with a 4-mL reaction module, and the assembled apparatus was placed onto a standard laboratory stir plate. Six reaction vials were inserted around the module center and held $7 \mathrm{~mm}$ above the photon source. The apparatus was turned on by plugging the WPP device into a standard 12 Volt 2 Amp power supply, and the reaction mixture was left to stir for the desired time.

Detailed Instructions for configuration, documentation and operation of WPP devices are provided within the separate fabrication and operation guide provided alongside this document. All component design files are provided in the project repository provided alongside this document. 
The following tables summarize photon source information and reaction module vessel information for this study.

Table S1: Summary of WPP photon sources used in the present study.

\begin{tabular}{cccc}
$\begin{array}{c}\text { LED } \\
\text { Color }\end{array}$ & $\begin{array}{c}\text { Max } \\
\text { Emission } \\
\text { Wavelength }\end{array}$ & $\begin{array}{c}\text { LED Manufacturer } \\
\text { and Part Number }\end{array}$ & $\begin{array}{c}\text { LEDSupply } \\
\text { LED Star } \\
\text { Part Number }\end{array}$ \\
\hline UVA & 368 & Inolux IN-C39CTOU2 & N/A (User mounted) \\
Violet & 395 & Inolux IN-C39CTOU5 & N/A (User mounted) \\
Blue & 448 & Cree, Inc. XTEARY-00-0000-000000N09 & CREEXTE-ROY-3 \\
Cyan & 495 & Luxeon LXML-PE01-0080 & 07007-PE000-K \\
Green & 528 & Cree, Inc. XPEBGR-L1-0000-00D01 & CREEXPE2-GRN-3 \\
Amber & 597 & Luxeon LXML-PL01-0060 & 07007-PL000-F \\
Orange & 620 & Cree, Inc. XPEBRO-L1-0000-00901 & CREEXPE2-RDO-3 \\
Red & 658 & Cree, Inc. XPEPHR-L1-0000-00801 & CREEXPE-DRD-3 \\
NIR & 731 & Cree, Inc. XPEFAR-L1-0000-00601 & CREEXPE-FRD-3
\end{tabular}

Table S2: Summary of reaction vessels used in the present study.

$\begin{array}{cc}\begin{array}{c}\text { Reaction } \\ \text { Vessel Size }\end{array} & \begin{array}{c}\text { Vessel Manufacturer } \\ \text { and Part Number }\end{array} \\ 2 \mathrm{~mL} & \text { Fisher Scientific 03-338AA } \\ 4 \mathrm{~mL} & \text { Chemglass CG-4909-04 } \\ 8 \mathrm{~mL} & \text { Chemglass CG-4909-03 } \\ 24 \mathrm{~mL} & \text { Vial: VWR 66012-044 } \\ & \text { Cap: Chemglass CG-4911-20 }\end{array}$




\subsection{Comparison Setups}
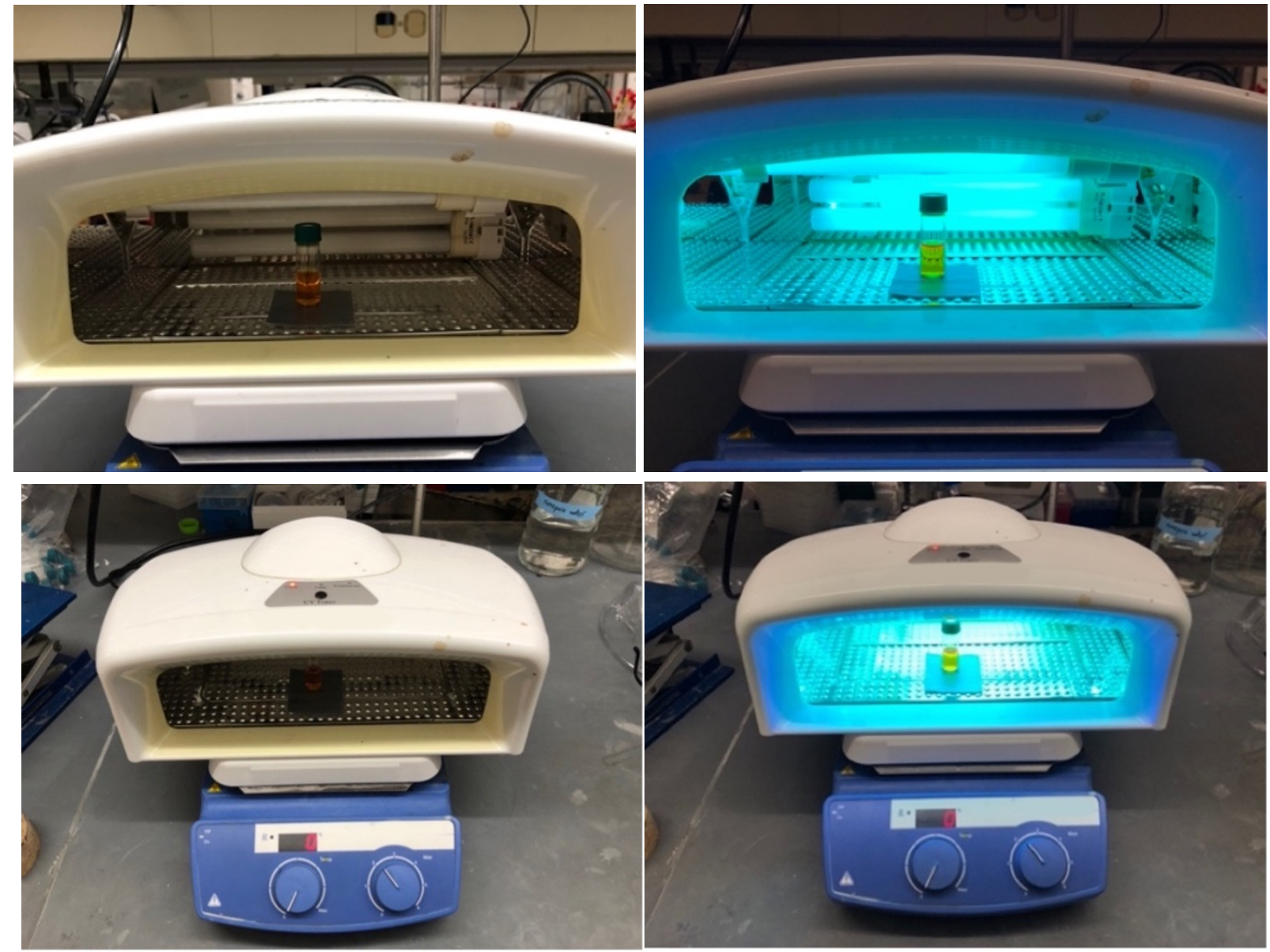

Figure S3: UV curing chamber photoreactor setup

Benchmarking experiment procedure: A Thermal Spa nail dryer UV curing chamber (Amazon Standard Identification Number [ASIN]: B001RMP7M6) was placed onto a standard laboratory stir plate, and a $4 \mathrm{~mL}$ reaction vial was inserted into the center of the chamber ca. $7.5 \mathrm{~cm}$ deep. Multiple layers of duct tape were placed beneath the vial to provide a flat surface for the reaction vessel to stand on while stirring. A desktop fan (not shown) was placed in front of the apparatus to provide cooling. The apparatus was switched on, set to "continuous mode," and the reaction solution was allowed to stir for the desired time.

This setup is based upon the inexpensive UV photoreactor detailed in reference 1. 

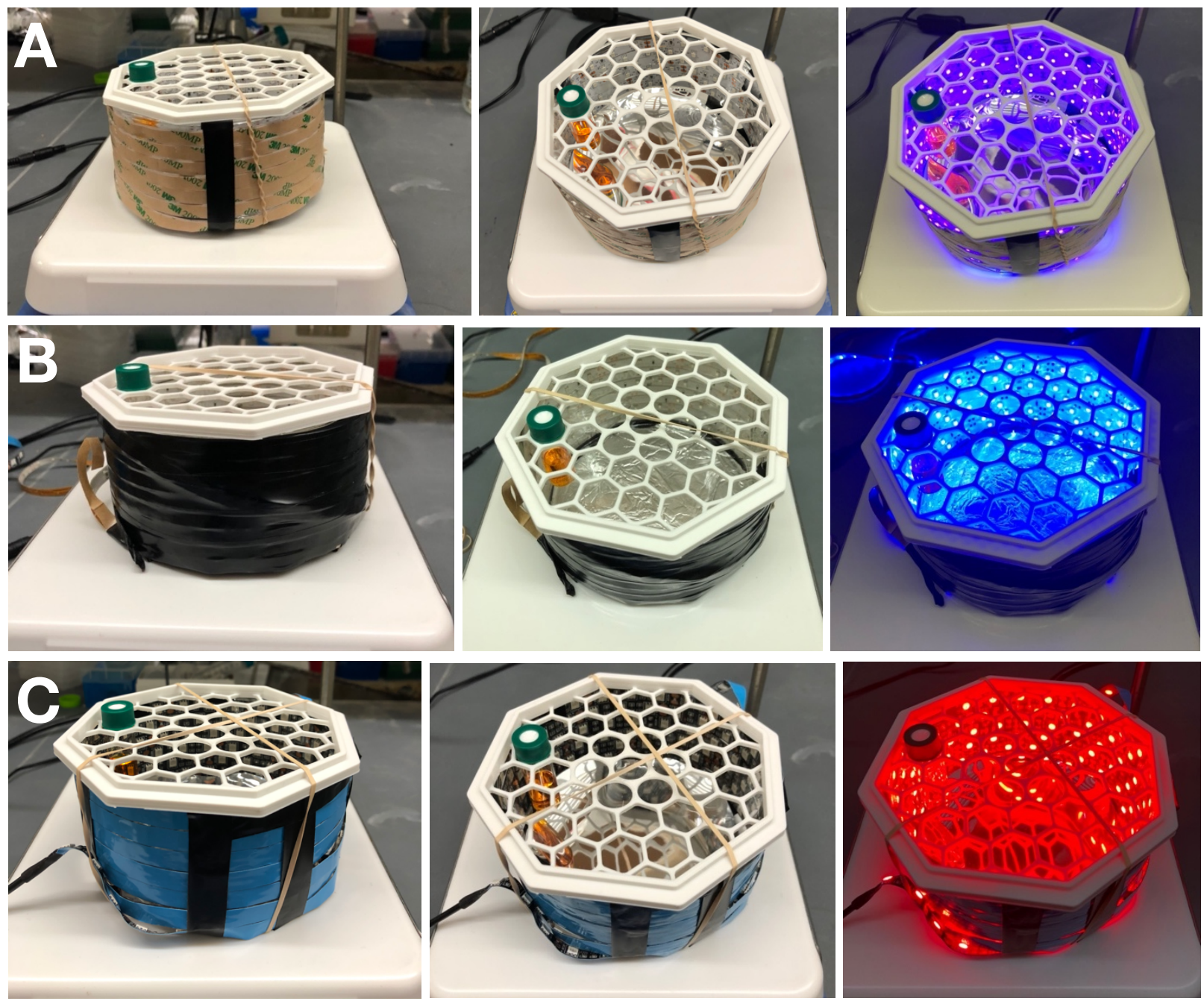

Figure S4: (A) $400 \mathrm{~nm}$, (B) $450 \mathrm{~nm}$ and (C) $630 \mathrm{~nm}$ LED strip photoreactor setups

Benchmarking experiment procedure: The outside of a $700 \mathrm{~mL}$ VWR crystallizing dish was wrapped 7-8 times with a commercial 24 W 400 nm (ASIN: B07VBL7GSZ), 24 W 450 nm (ASIN: B07D7SSVCQ) or $38 \mathrm{~W} 630 \mathrm{~nm}$ (ASIN: B07QMLT395) LED strip and taped in place with electrical tape. A reflective piece of foil was placed in the bottom of the dish, an inverted 4-mL vessel holder from a WPP device was placed on top of the dish, and the apparatus was secured with rubber bands. The assembled LED strip photoreactor was placed onto a standard laboratory stir plate, a $4 \mathrm{~mL}$ reaction vial was inserted into the vial holder ca. $1 \mathrm{~cm}$ away from the wall of the crystallizing dish, and a desktop fan (not shown) was placed above the apparatus to provide cooling. The apparatus was turned on by plugging the LED strip into a standard 12 Volt, 2 Amp power supply, and the reaction mixture was left to stir for the desired time.

This setup is based upon the batch photoreaction apparatus reported in references 2 and 4 . 

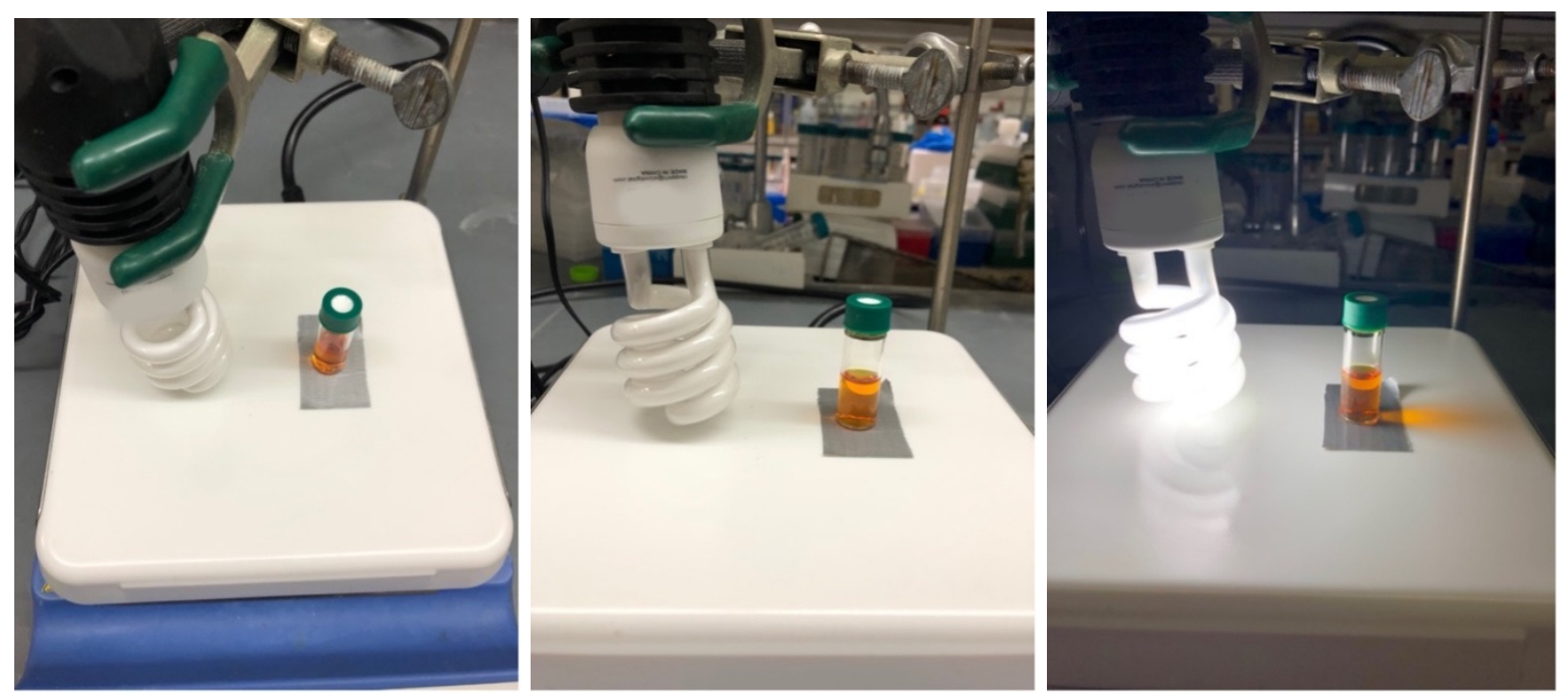

Figure S5: Compact fluorescent light (CFL) bulb photoreaction setup

Benchmarking experiment procedure: An ALZO digital full spectrum $15 \mathrm{~W}$ CFL bulb (ASIN: B0089MIH8I) was screwed into a switchable lightbulb socket and suspended above a standard laboratory stir plate using a lab clamp. A $4 \mathrm{~mL}$ reaction vial was placed on the stir plate ca. $4 \mathrm{~cm}$ away from the CFL bulb. A piece of duct tape was placed underneath the reaction vial to keep the vial in place while stirring. A desktop fan (not shown) was placed to the side of the apparatus to provide cooling. The CFL bulb was switched on, and the reaction mixture was left to stir for the desired time.

This photoreaction setup is based upon the household light bulb setup depicted in references 3 and 4. 

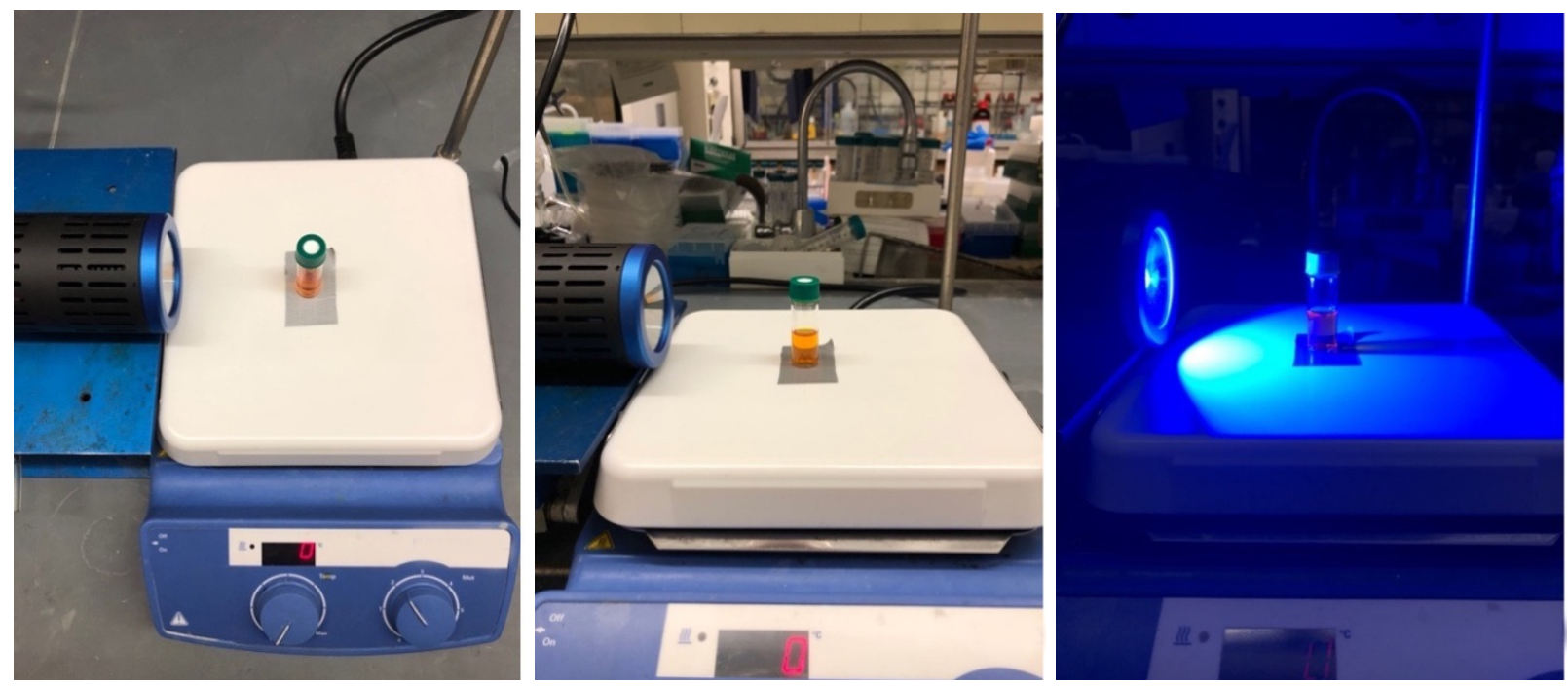

Figure S6: Kessil A160WE light emitting diode (LED) Lamp photoreaction setup

Benchmarking experiment procedure: A 40 W Kessil A160 WE Tuna Blue LED lamp (ASIN: B00QHC6D7O) was placed onto a laboratory jack ca. $1 \mathrm{~mm}$ higher than the top of a laboratory stir plate. A $4 \mathrm{~mL}$ reaction vial was placed on the stir plate ca. $7 \mathrm{~cm}$ away from the lamp. A piece of duct tape was placed beneath the reaction vial to keep the vial in place while stirring. A desktop fan (not shown) was placed to the side of the apparatus to provide cooling. The "blue" and "intensity" settings of the lamp were adjusted to maximum, the lamp was turned on by plugging it in and the reaction was left to stir for the desired time.

This photoreaction setup is based upon the standard LED lamp setup reported in reference 4 . 


\section{Photon Source Characterization}

\subsection{WPP Photon Source Emission Profiles}

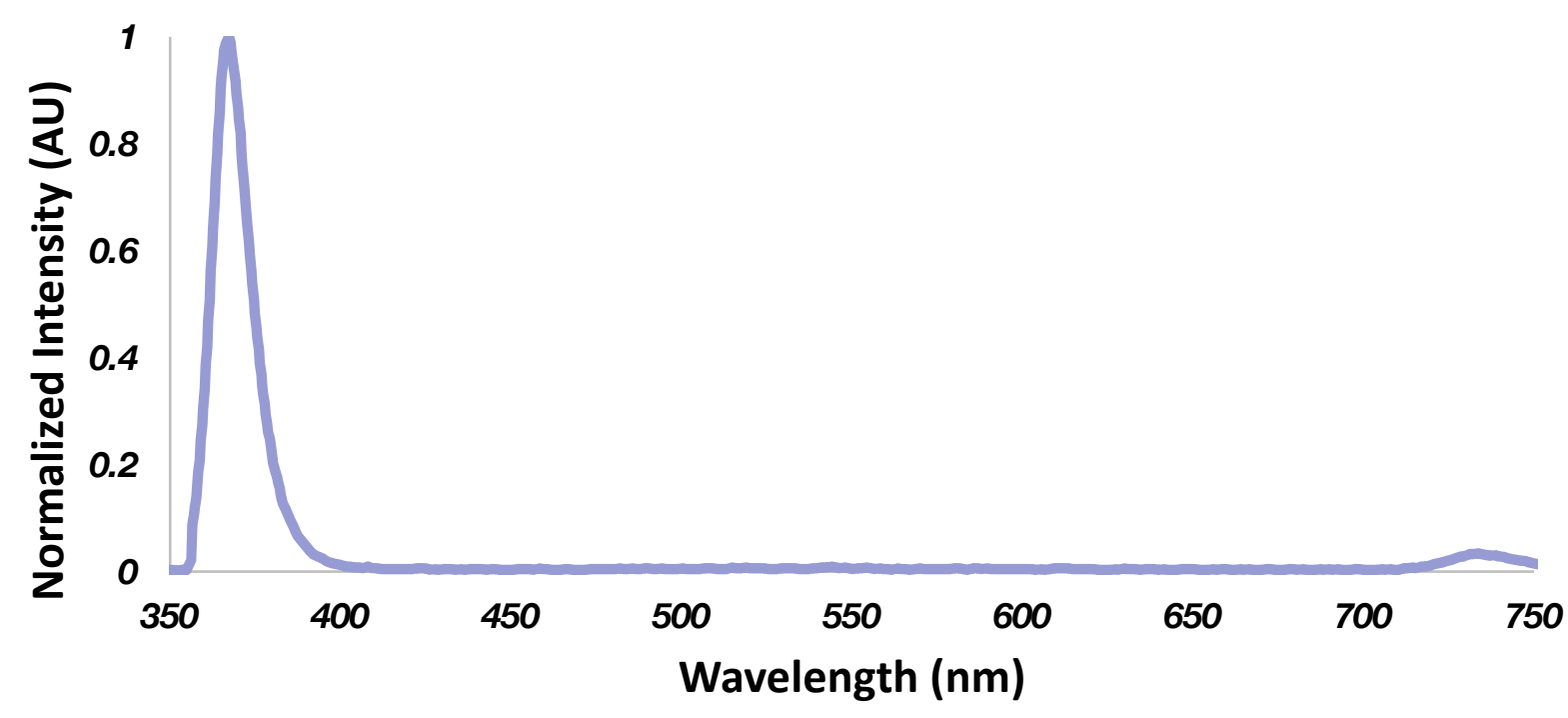

Manufacturer

Manufacturer Part Number

Max Emission Wavelength
Inolux

IN-C68QACTMU2

$368 \mathrm{~nm}$

Figure S7: Emission spectrum of commercial high-intensity ultraviolet A (UVA) LEDs

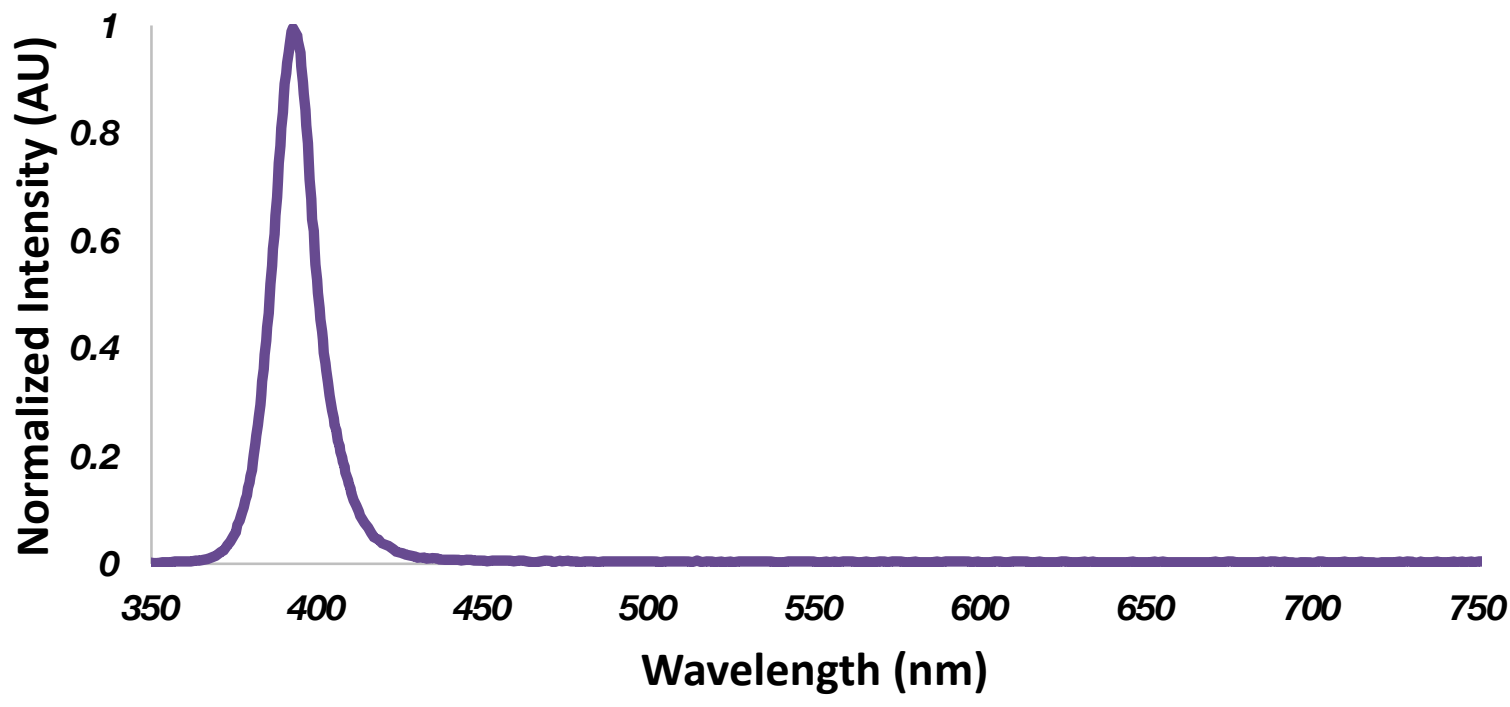

Manufacturer

Manufacturer Part Number

Max Emission Wavelength
Inolux

IN-C68QACTMU5

$395 \mathrm{~nm}$

Figure S8: Emission spectrum of commercial high-intensity violet LEDs 


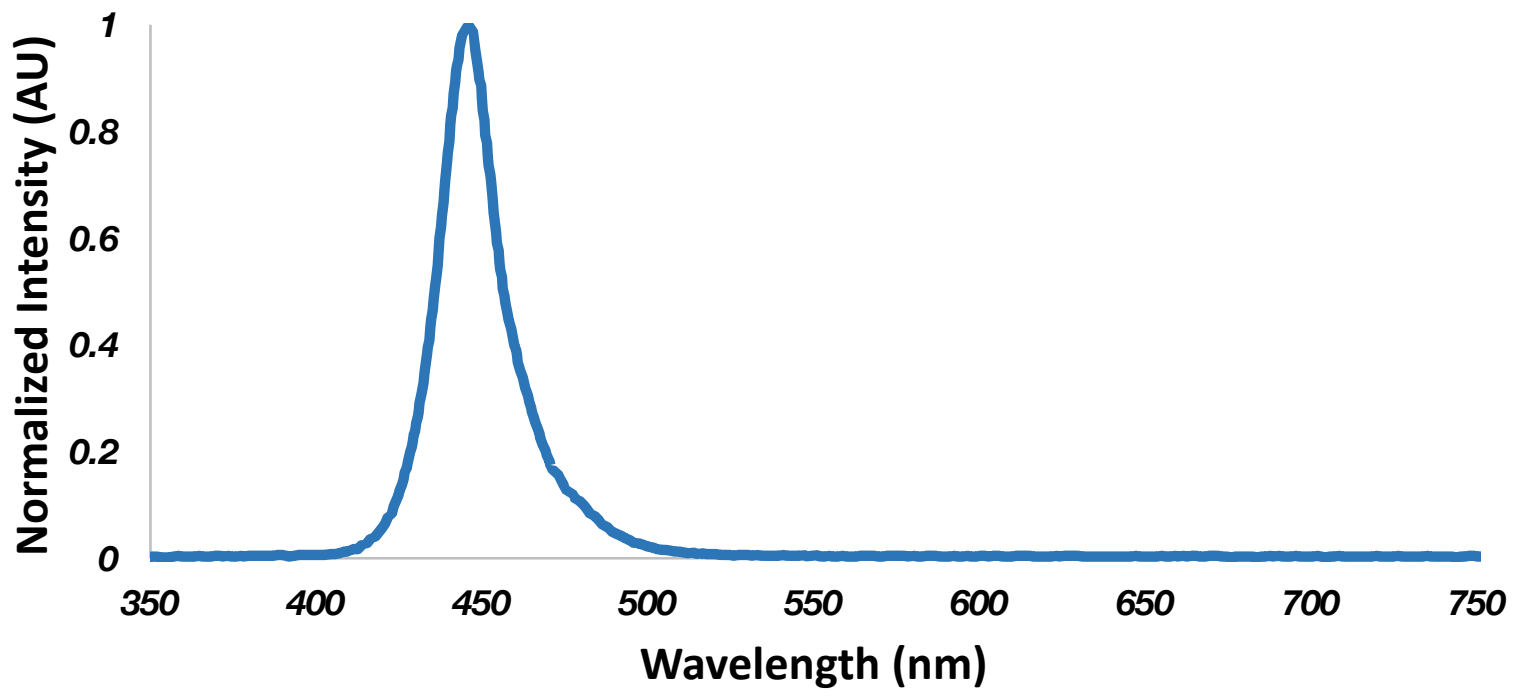

Manufacturer

Manufacturer Part Number

Max Emission Wavelength
Cree, Inc.

XTEARY-00-0000-000000N09

$448 \mathrm{~nm}$

Figure S9: Emission spectrum of commercial high-intensity blue LEDs

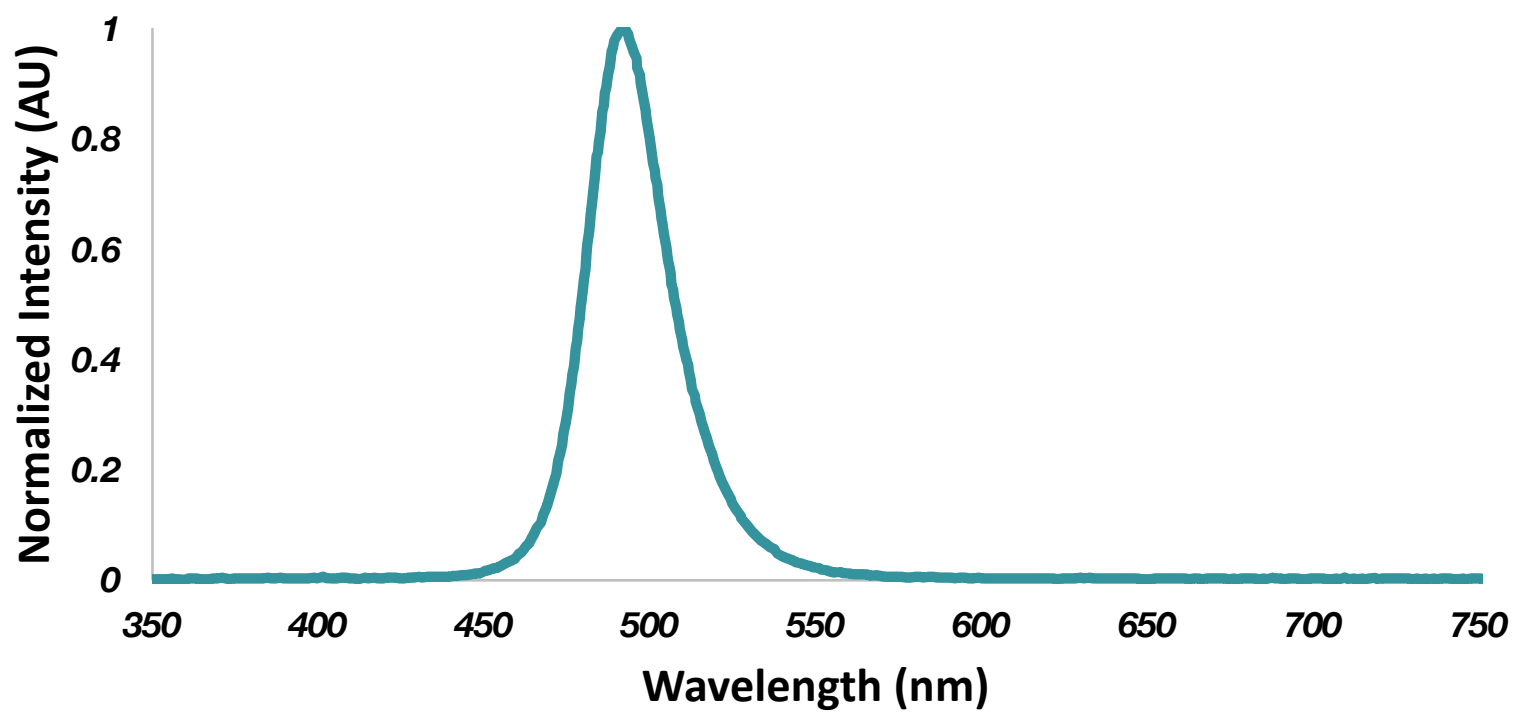

Manufacturer

Manufacturer Part Number

Max Emission Wavelength
Luxeon

LXML-PE01-0080

$495 \mathrm{~nm}$

Figure S10: Emission spectrum of commercial high-intensity cyan LEDs 


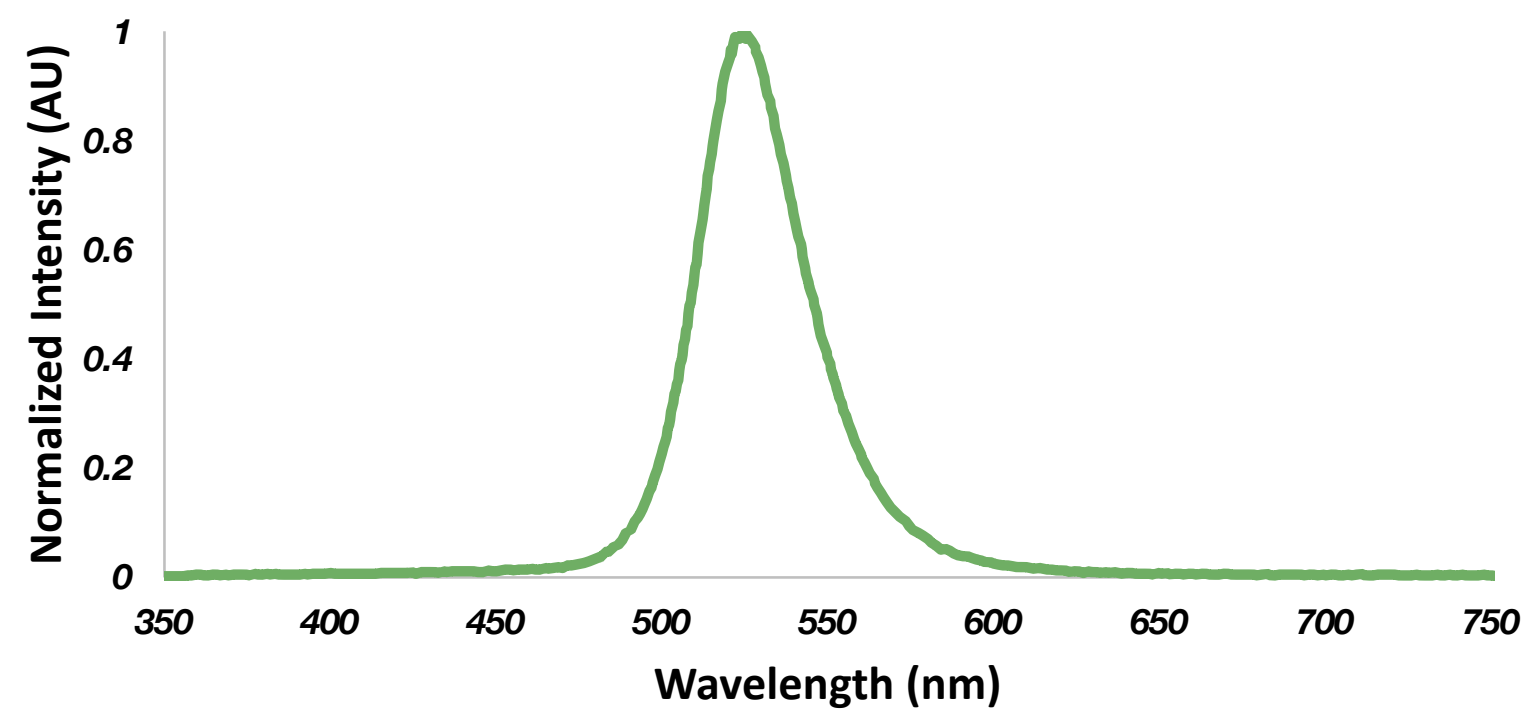

Manufacturer

Manufacturer Part Number

Max Emission Wavelength
Cree, Inc.

XPEBGR-L1-0000-00D01

$528 \mathrm{~nm}$

Figure S11: Emission spectrum of commercial high-intensity green LEDs

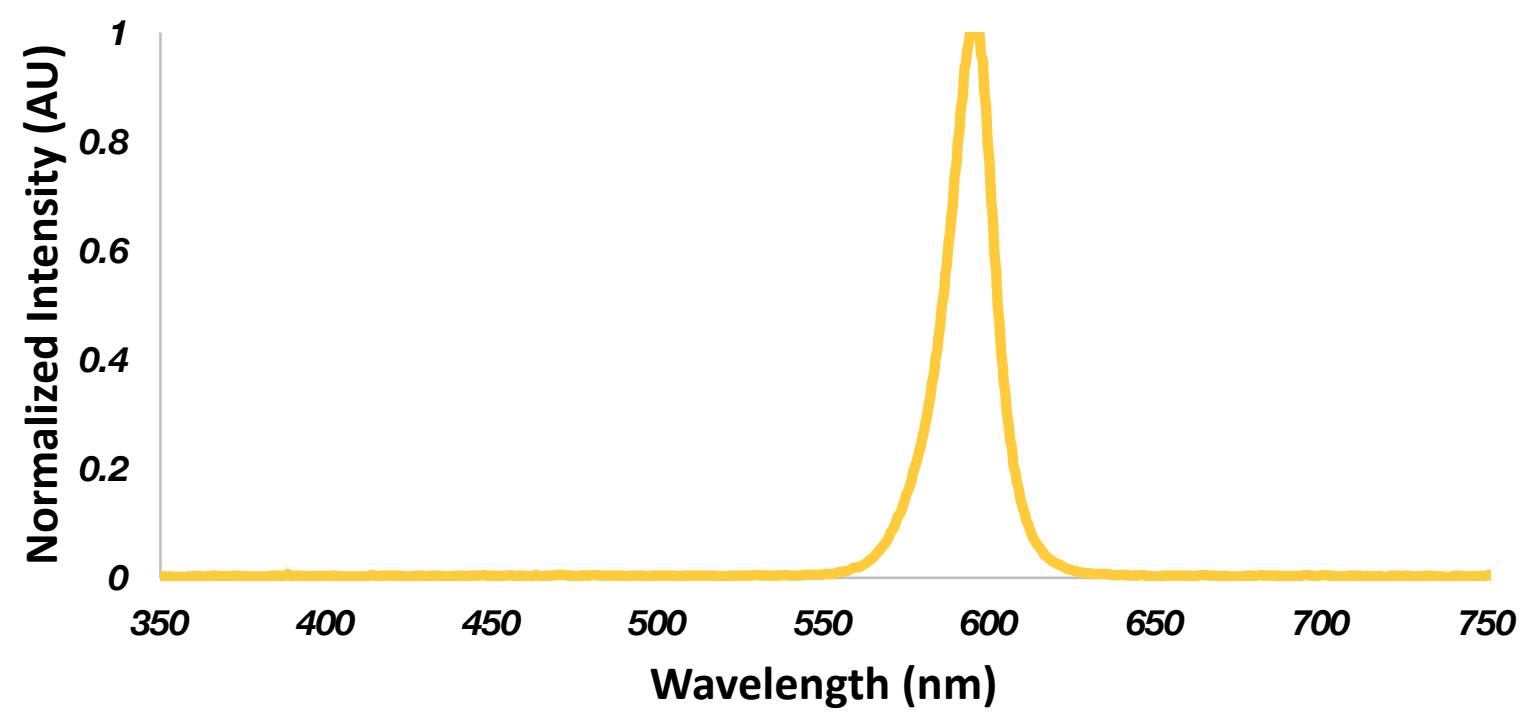

Manufacturer

Manufacturer Part Number

Max Emission Wavelength
Luxeon

LXML-PE01-0060

$597 \mathrm{~nm}$

Figure S12: Emission spectrum of commercial high-intensity amber LEDs 


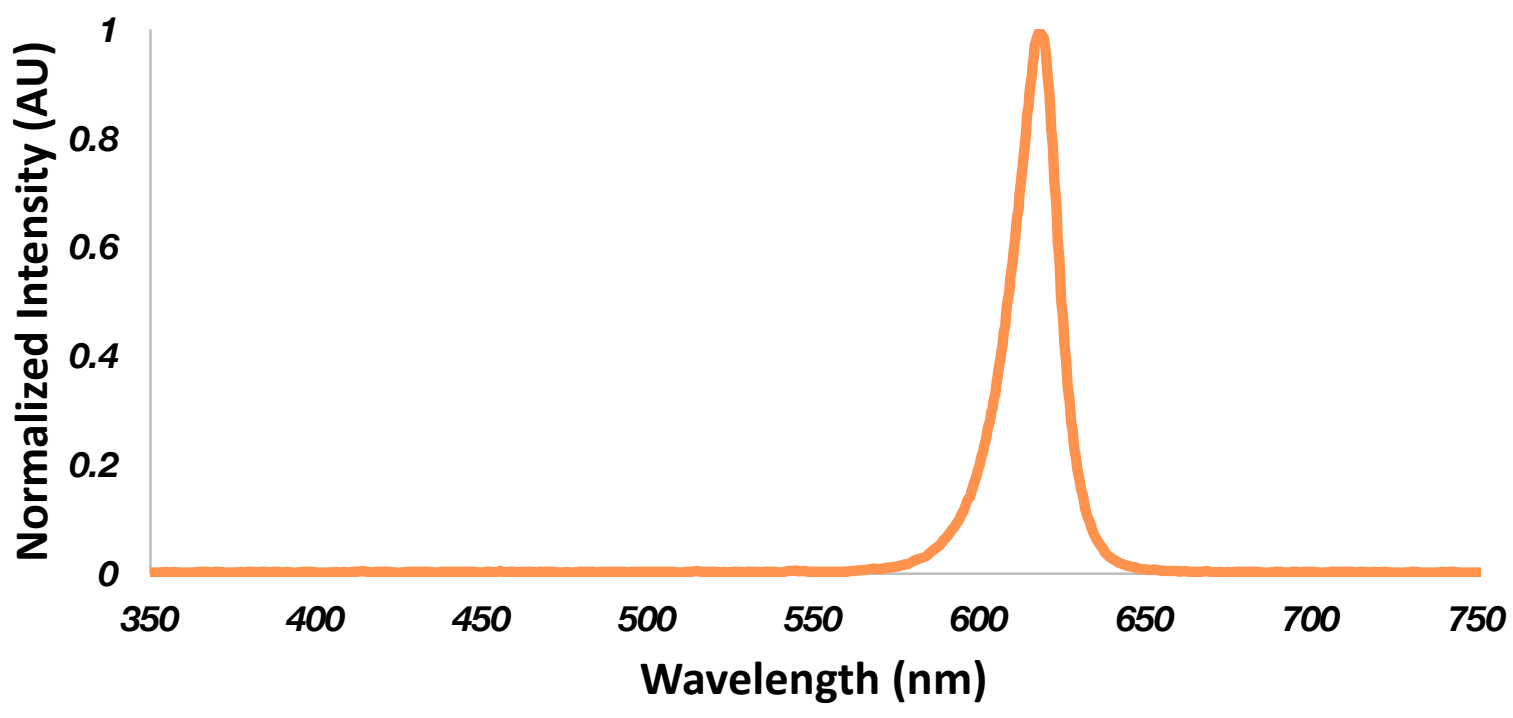

Manufacturer

Manufacturer Part Number

Max Emission Wavelength

Cree, Inc.

XPEBRO-L1-0000-00901

$620 \mathrm{~nm}$

Figure S13: Emission spectrum of commercial high-intensity orange LEDs

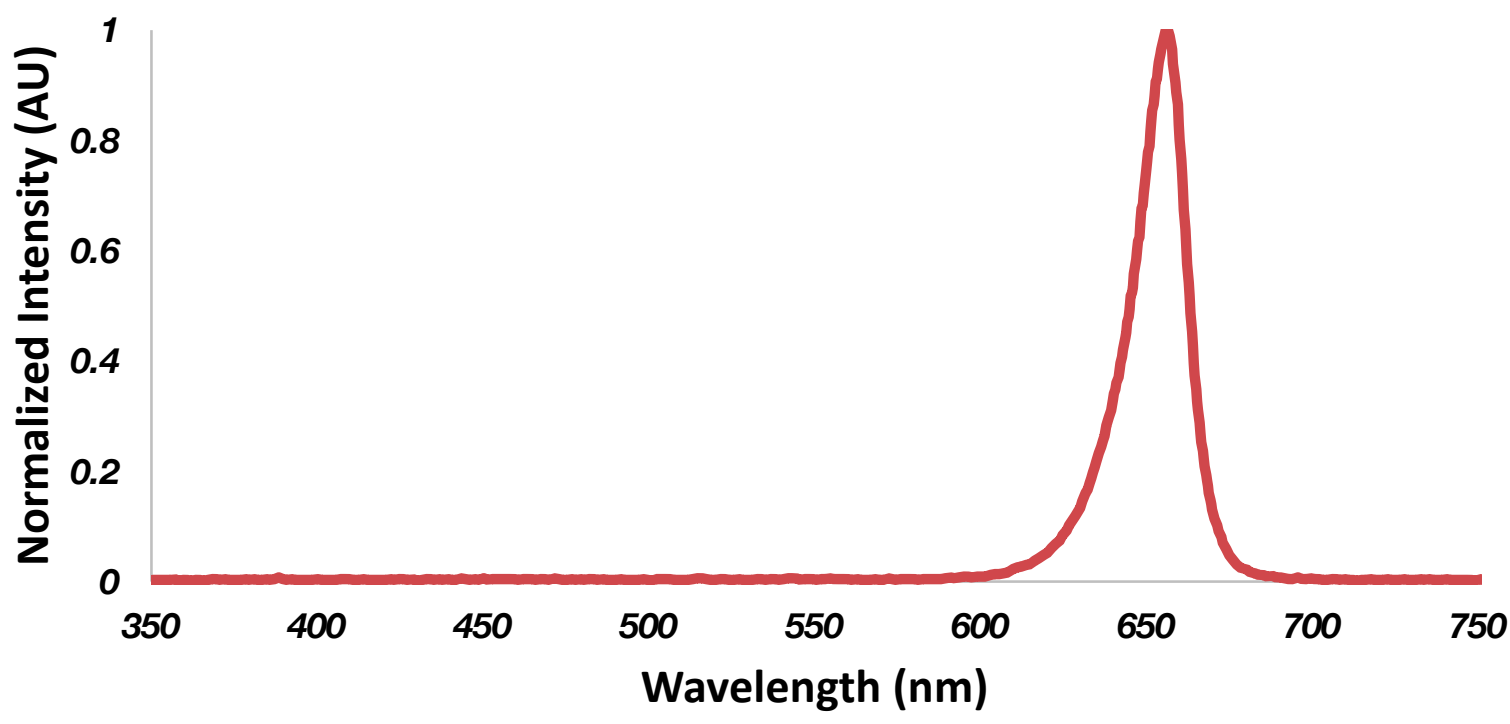

Manufacturer

Manufacturer Part Number

Max Emission Wavelength
Cree, Inc.

XPEPHR-L1-0000-00801

$658 \mathrm{~nm}$

Figure S14: Emission spectrum of commercial high-intensity red LEDs 


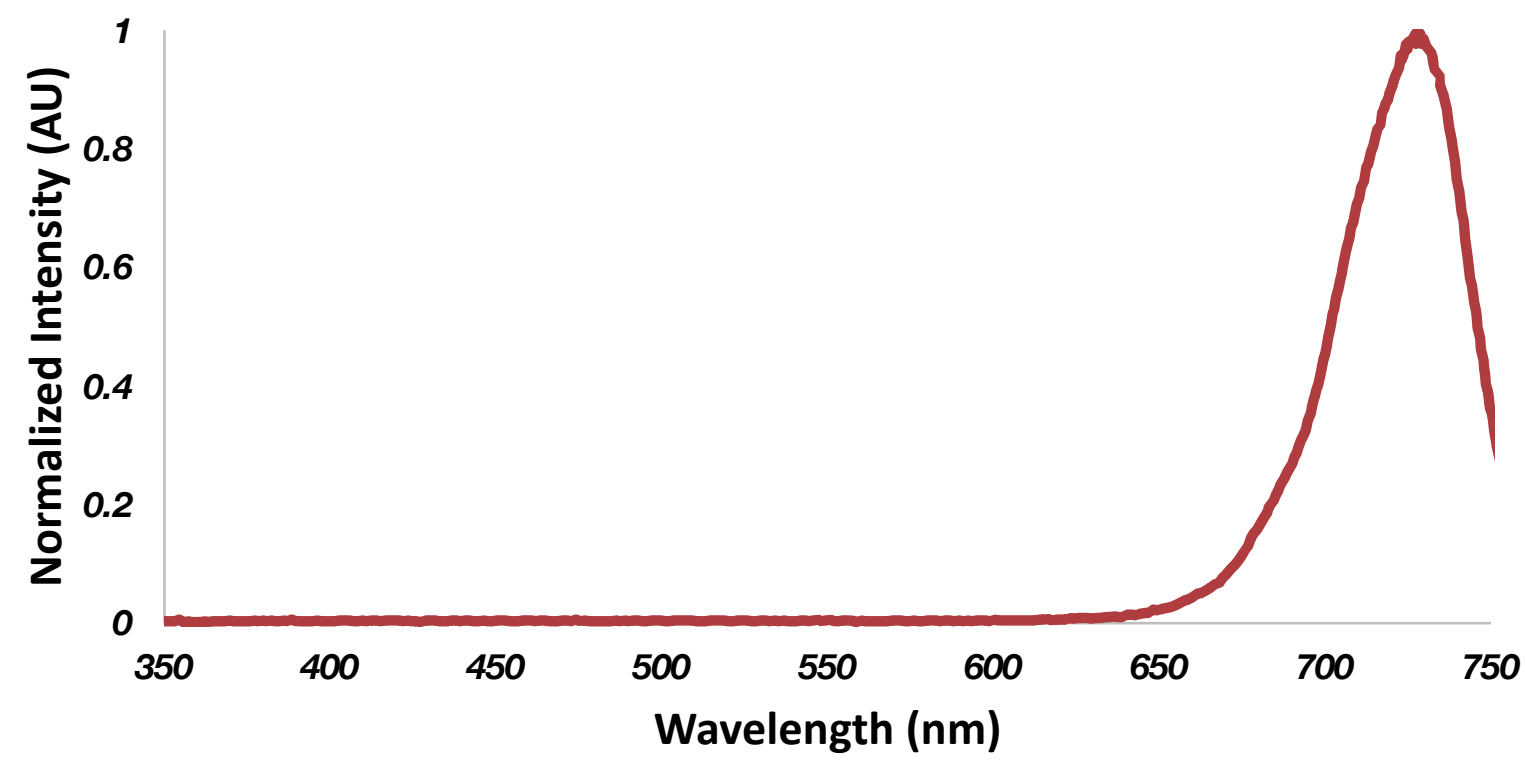

Manufacturer

Manufacturer Part Number

Max Emission Wavelength
Cree, Inc.

XPEFAR-L1-0000-00601

$731 \mathrm{~nm}$

Figure S15: Emission spectrum of commercial high-intensity near infra-red (NIR) LEDs

\subsection{Comparison Setups Emission Profiles}

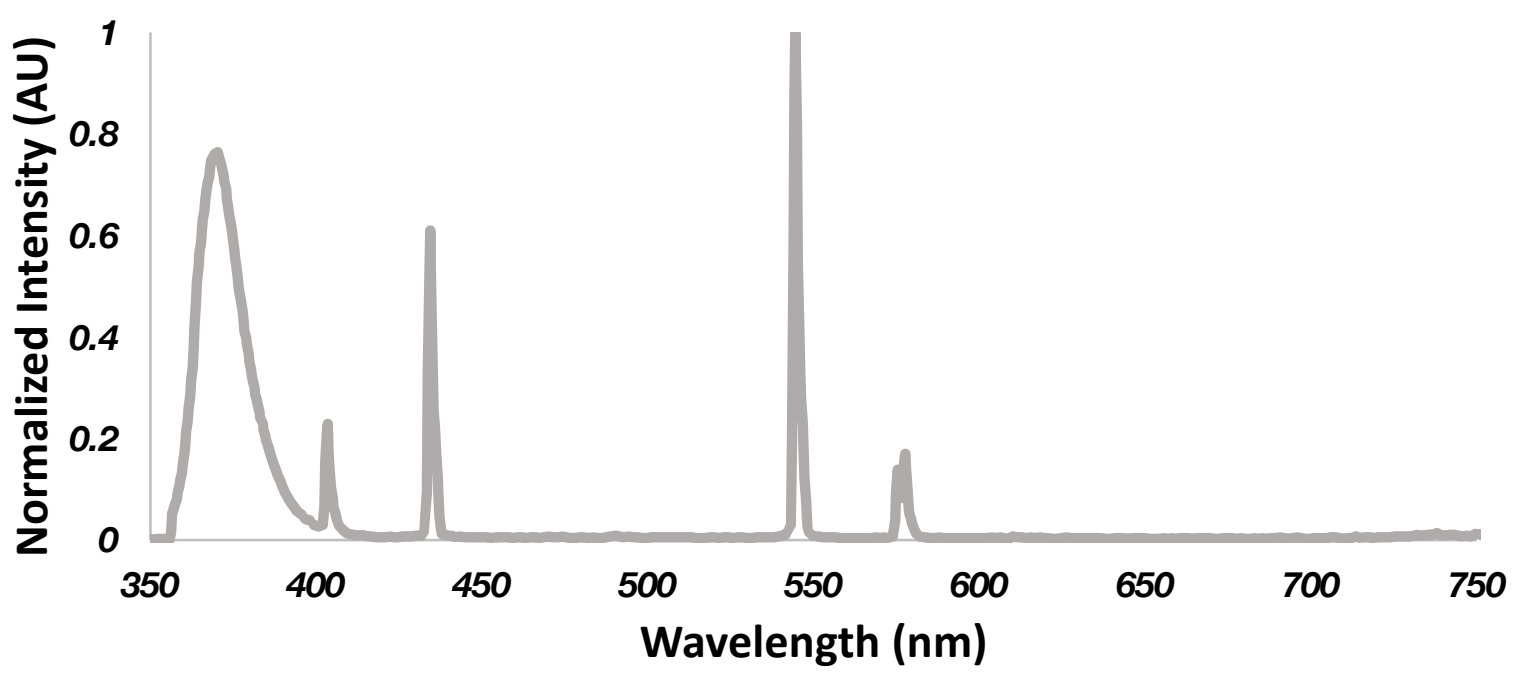

\section{Manufacturer ASIN \\ Max Emission Wavelength}

Thermal Spa B001RMP7M6

$545 \mathrm{~nm}$

Figure S16: Emission spectrum of UV curing chamber photoreactor 


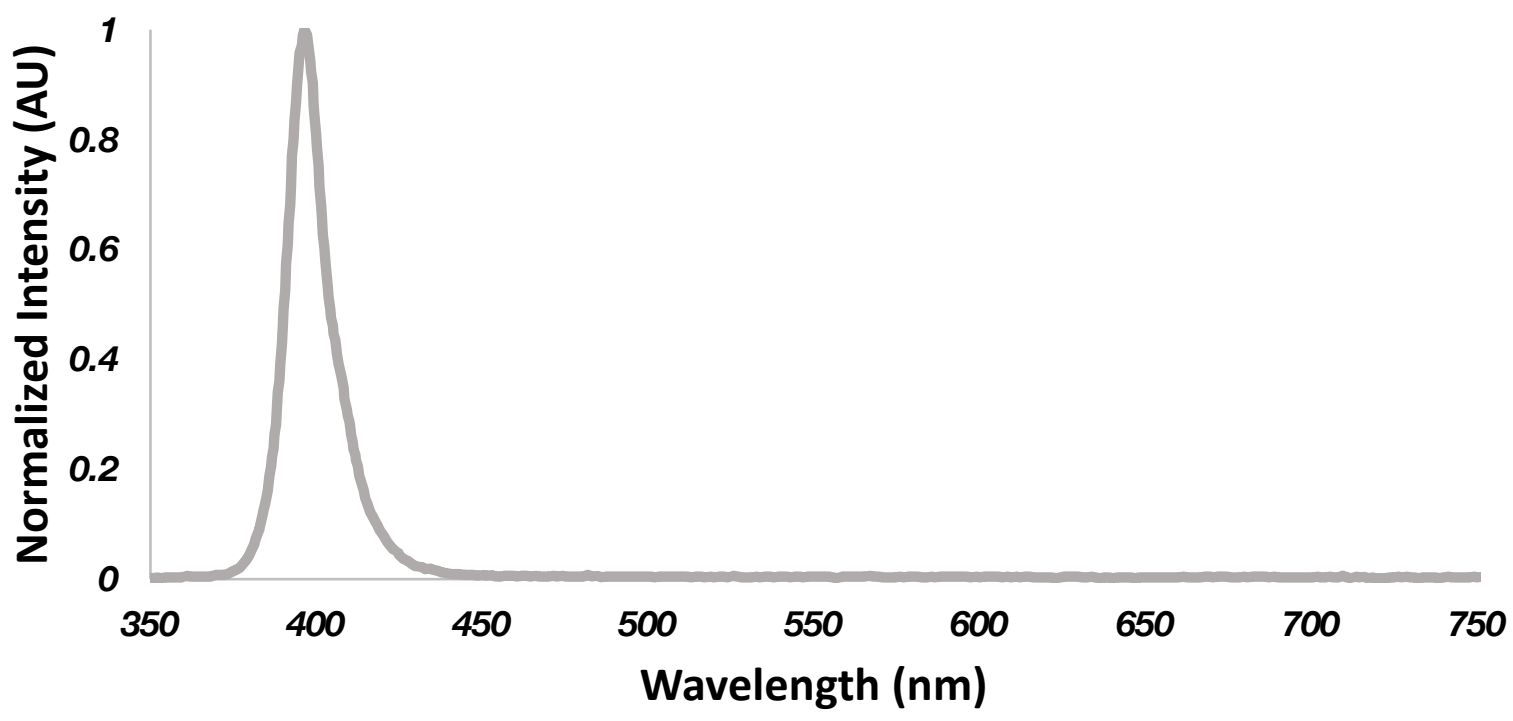

Manufacturer ASIN

Max Emission Wavelength
Sunmerit

B07VBL7GSZ

$398 \mathrm{~nm}$

Figure S17: Emission spectrum of $400 \mathrm{~nm}$ LED strip

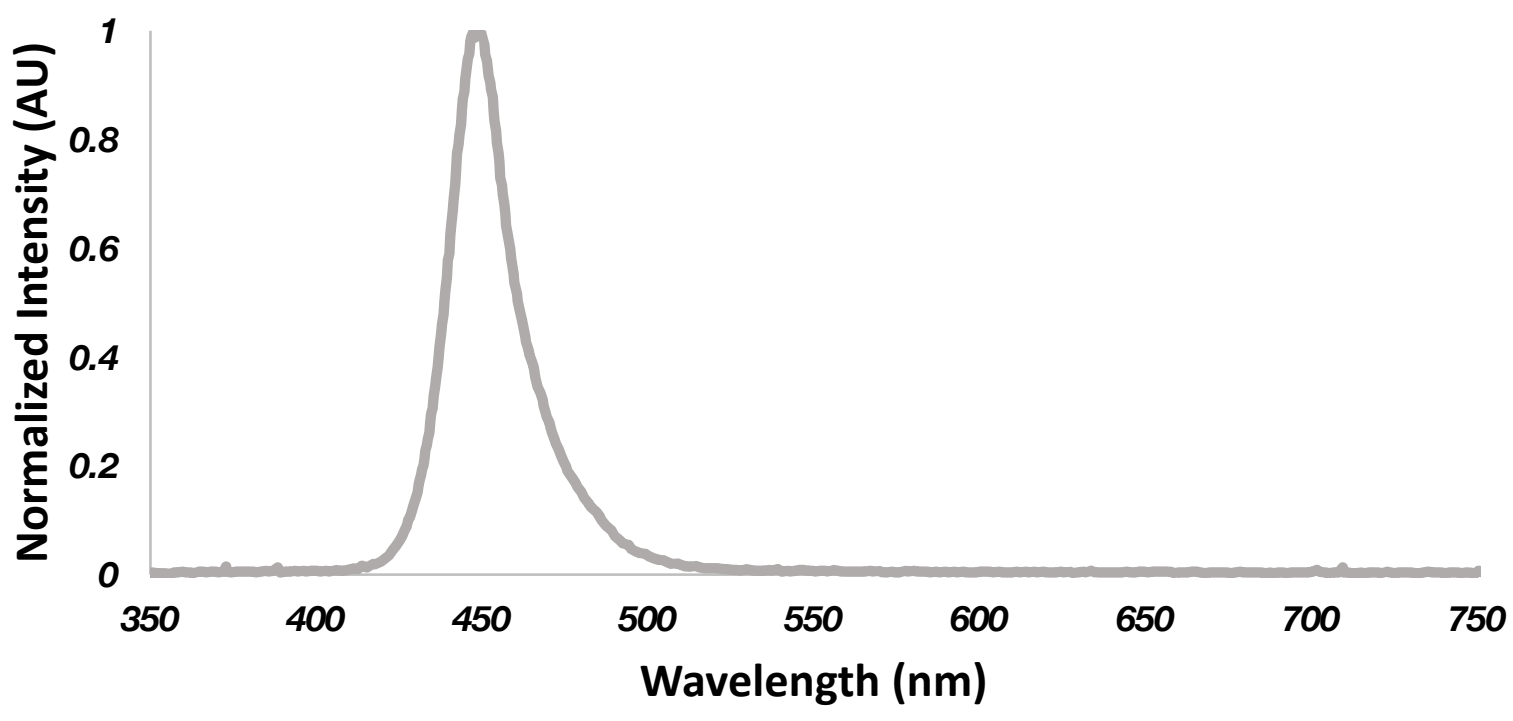

Manufacturer

ASIN

Max Emission Wavelength
XINKAITE

B07D7SSVCQ

$451 \mathrm{~nm}$

Figure S18: Emission spectrum of $450 \mathrm{~nm}$ LED strip 


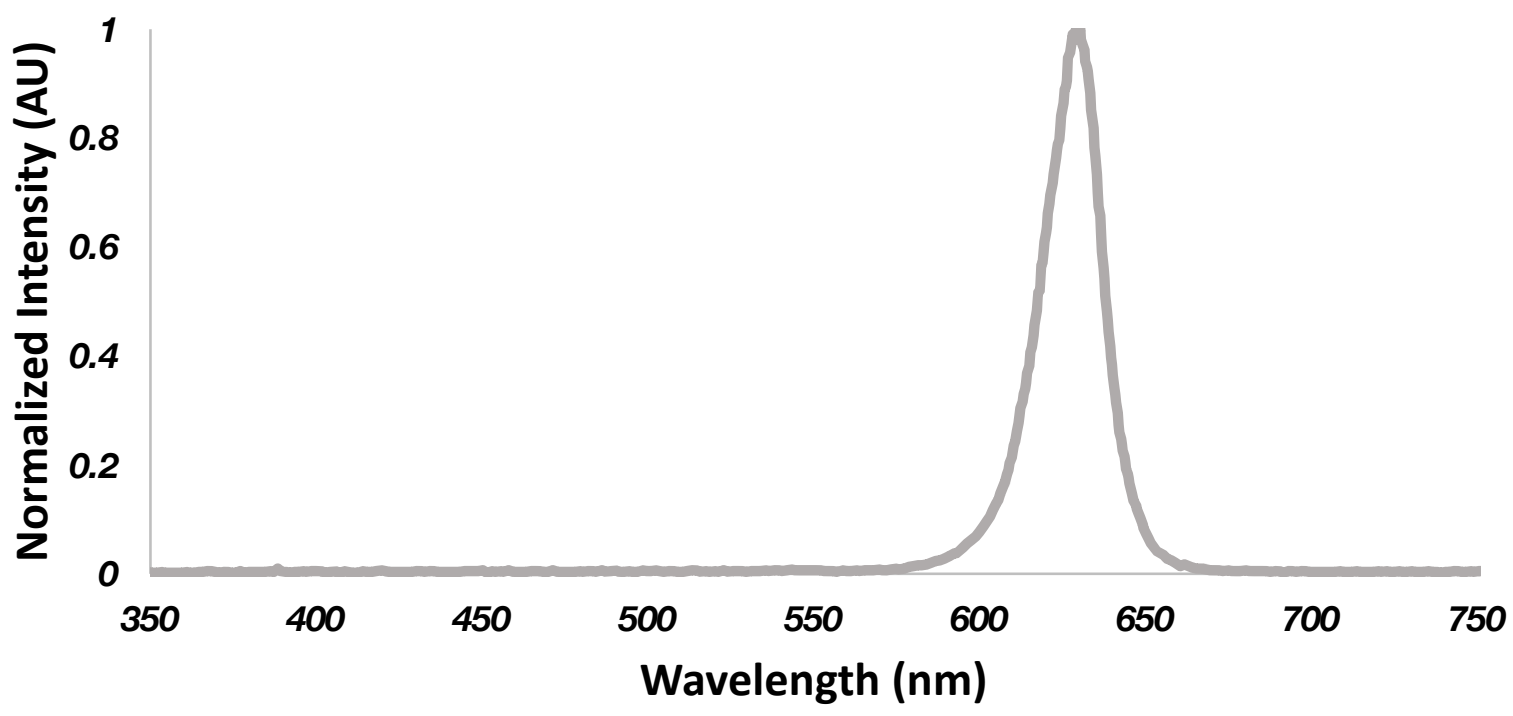

Manufacturer

ASIN

Max Emission Wavelength
YUNBO

B07QMLT395

$632 \mathrm{~nm}$

Figure S19: Emission spectrum of 630 nm LED strip

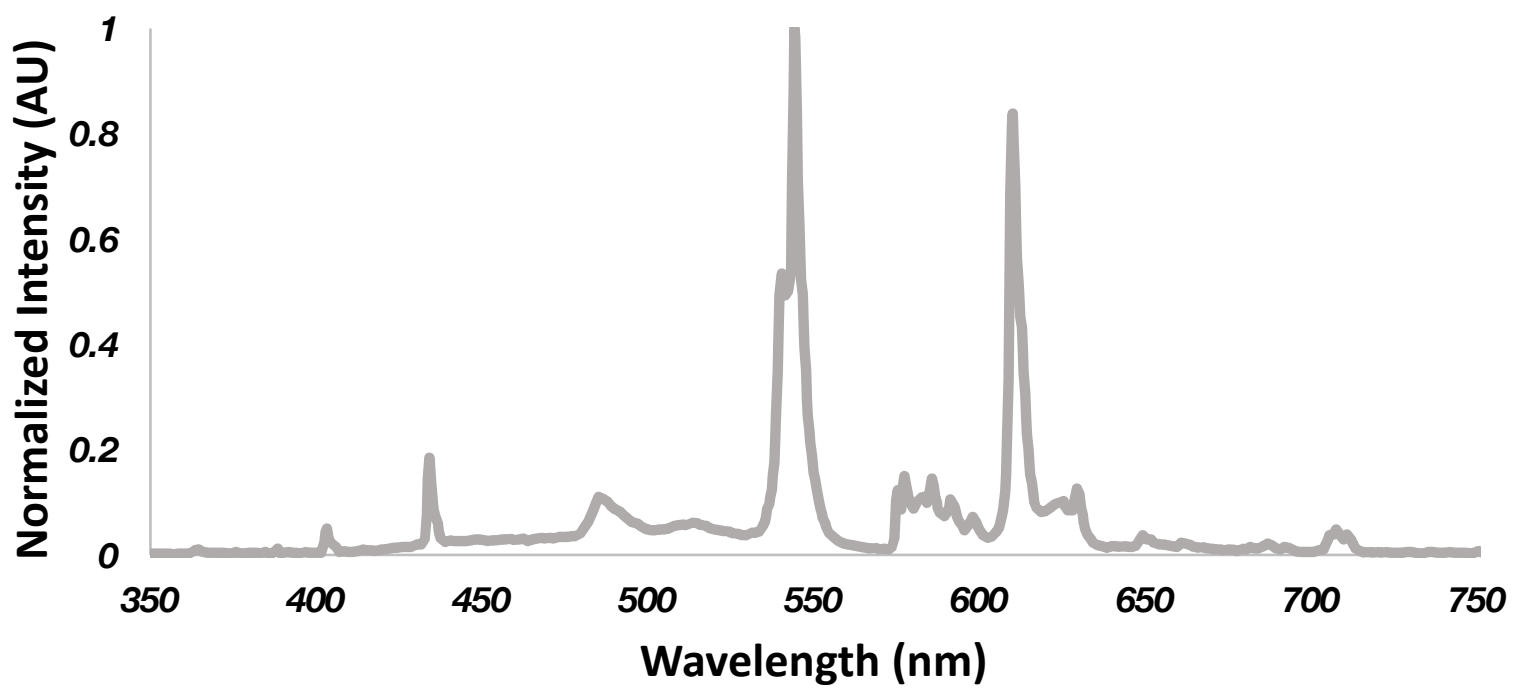

Manufacturer

ASIN

Max Emission Wavelength
ALZO Digital

B0089MIH8I

$545 \mathrm{~nm}$

Figure S20: Emission spectrum of CFL bulb 


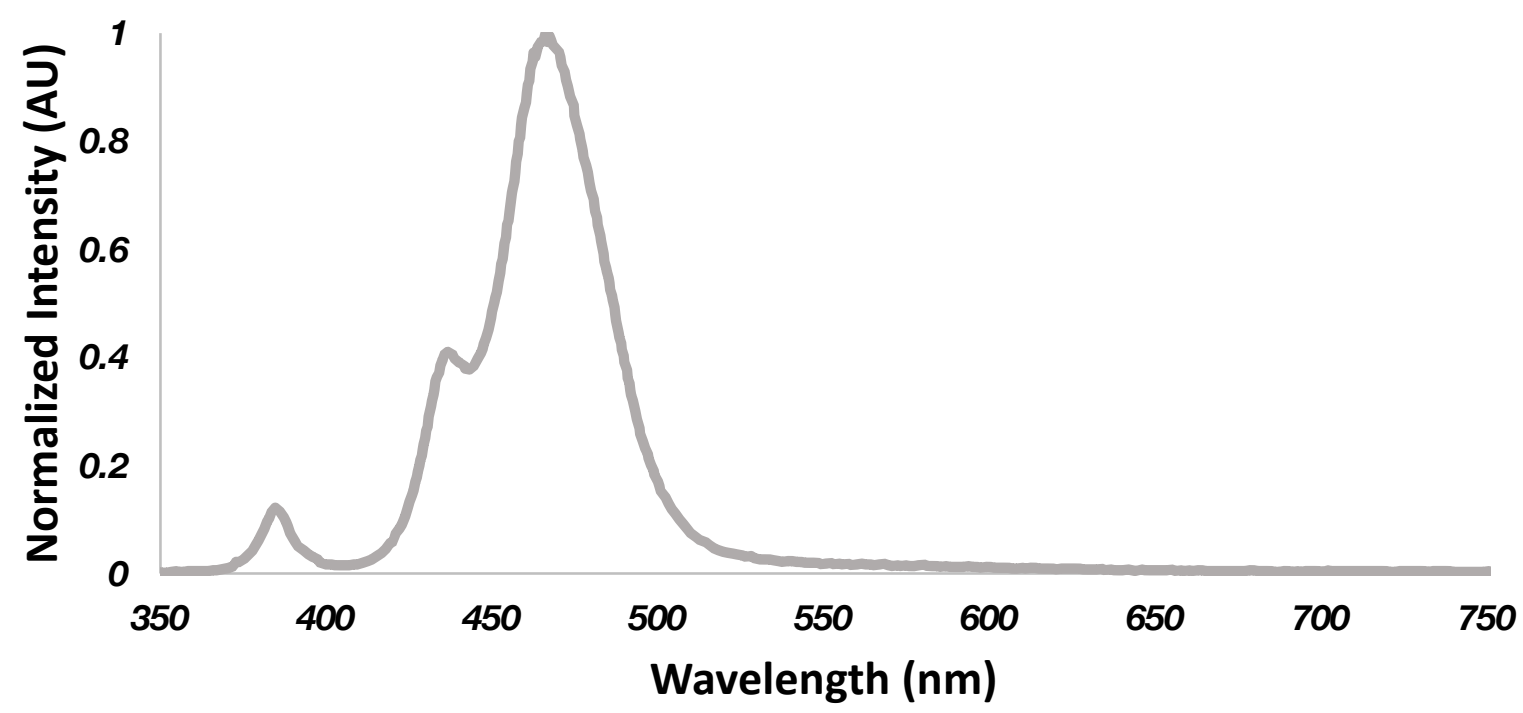

$\begin{array}{cc}\text { Manufacturer } & \text { Kessil } \\ \text { ASIN } & \text { B00QHC6D7O } \\ \text { Max Emission Wavelength } & 451 \mathrm{~nm}\end{array}$

Figure S21: Emission spectrum of Kessil A160WE LED lamp 


\section{Experimental Procedures}

\subsection{Benchmark 1 - C-N Cross-coupling of 4-Bromobenzotrifluoride and Morpholine via Photoexcitation of Ni-amine Complexes}

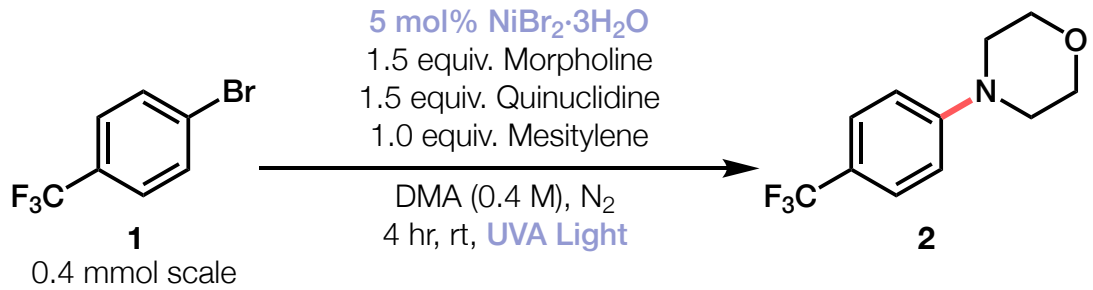

Procedure: To a $4 \mathrm{~mL}$ vial equipped with a stir bar was added quinuclidine $(66.7 \mathrm{mg}, 0.6 \mathrm{mmol}$, 1.5 eq.) under air. The vessel was capped, placed under $\mathrm{N}_{2}$ and charged with a solution of $\mathrm{NiBr}_{2} \cdot 3 \mathrm{H}_{2} \mathrm{O}$ (5.5 mg, $0.02 \mathrm{mmol}, 0.05$ eq.) in dry DMA (1 mL). To the vial was then added morpholine $(51.7 \mu \mathrm{L}, 0.6 \mathrm{mmol}, 1.5$ eq.) and 4-bromobenzotrifluoride (56.0 $\mu \mathrm{L}, 0.4 \mathrm{mmol}, 1.0$ eq.). The resulting solution was sparged with $\mathrm{N}_{2}$ for 30 minutes at $0{ }^{\circ} \mathrm{C}$. The reaction vessel was warmed to room temperature, and mesitylene $(55.6 \mu \mathrm{L}, 0.4 \mathrm{mmol}, 1.0$ eq.) was added as an internal standard. The vessel was wrapped with parafilm, placed into a UVA light photoreaction setup and illuminated while stirring for 4 hours. Reaction progress was monitored using ${ }^{1} \mathrm{H}$ NMR analysis of reaction aliquots diluted with DMSO- $d_{6}$. Crude product was purified using previously reported methods yielding a white amorphous solid $(98.0 \mathrm{mg}, 94 \%$ isolated yield). Isolated product matched literature values. ${ }^{5}$

${ }^{1} \mathrm{H}$ NMR (600 MHz, CDCl $): \delta 7.50(\mathrm{~d}, \mathrm{~J}=8.7 \mathrm{~Hz}, 2 \mathrm{H}), 6.92(\mathrm{~d}, \mathrm{~J}=8.5 \mathrm{~Hz}, 2 \mathrm{H}), 3.86(\mathrm{t}, \mathrm{J}=5.0 \mathrm{~Hz}$, $4 \mathrm{H}) 3.23(\mathrm{t}, \mathrm{J}=5.0 \mathrm{~Hz}, 4 \mathrm{H}) \mathrm{ppm}$.

${ }^{13} \mathrm{C}$ NMR (151 MHz, CDCl $): \delta 153.5,127.5,126.6$ (q, $\left.\mathrm{J}_{C-F}=3.8\right), 124.7$ (q, $\left.\mathrm{J}_{C-F}=270 \mathrm{~Hz}\right), 121.1$ (q, $\left.\mathrm{J}_{\mathrm{C}-\mathrm{F}}=33 \mathrm{~Hz}\right), 114.4,66.8,48.3 \mathrm{ppm}$.

$\left.{ }^{19} \mathrm{~F} \mathrm{NMR} \mathrm{(376} \mathrm{MHz,} \mathrm{CDCl}_{3}\right): \delta-61.4(3 \mathrm{~F}) \mathrm{ppm}$.

Table S3: Percent yield by ${ }^{1} \mathrm{H}$ NMR for different photoreaction setups taken over the time course of benchmark 1.

\begin{tabular}{|c|c|c|c|c|c|c|c|c|c|c|}
\hline $\begin{array}{c}0 \\
\min \end{array}$ & $\begin{array}{c}5 \\
\min \end{array}$ & $\begin{array}{c}10 \\
\min \end{array}$ & $\begin{array}{c}20 \\
\text { min }\end{array}$ & $\begin{array}{c}30 \\
\text { min }\end{array}$ & $\begin{array}{c}45 \\
\text { min }\end{array}$ & $\begin{array}{c}60 \\
\min \end{array}$ & $\begin{array}{c}90 \\
\min \end{array}$ & $\begin{array}{l}120 \\
\min \end{array}$ & $\begin{array}{l}180 \\
\mathrm{~min}\end{array}$ & $\begin{array}{l}240 \\
\text { min }\end{array}$ \\
\hline $0 \%$ & $9 \%$ & $18 \%$ & $34.5 \%$ & $48 \%$ & $61.5 \%$ & $73.5 \%$ & $88.5 \%$ & $96 \%$ & $96 \%$ & $96 \%$ \\
\hline $1.5 \%$ & $4.5 \%$ & $9 \%$ & $16.5 \%$ & $24 \%$ & $34.5 \%$ & $43.5 \%$ & $58.5 \%$ & $69 \%$ & $87 \%$ & $96 \%$ \\
\hline $0 \%$ & $0 \%$ & $1.5 \%$ & $4.5 \%$ & $9 \%$ & $13.5 \%$ & $18 \%$ & $25.5 \%$ & $33 \%$ & $43.5 \%$ & $52.5 \%$ \\
\hline
\end{tabular}




\subsection{Benchmark 2 - Vitamin-catalyzed Synthesis of 3,4-Benzocoumarin}

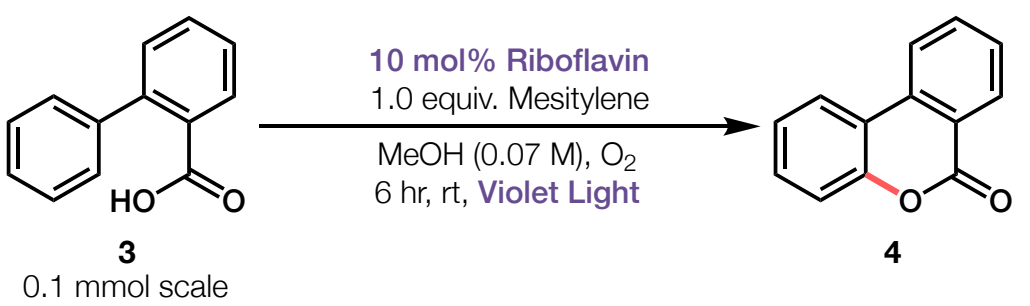

Procedure: To a $4 \mathrm{~mL}$ vial equipped with a stir bar were added riboflavin $(3.8 \mathrm{mg}, 0.01 \mathrm{mmol}$, 0.1 eq.), biphenyl-2-carboxylic acid (19.8 mg, 0.1 mmol, 1.0 eq.) and $\mathrm{MeOH}(1.5 \mathrm{~mL}, 0.066 \mathrm{M}$ ) under air. The vessel was capped and sparged with $\mathrm{O}_{2}$ for 5 minutes at $0{ }^{\circ} \mathrm{C}$. The reaction vessel was then warmed to room temperature and mesitylene ( $13.9 \mu \mathrm{L}, 0.1 \mathrm{mmol}, 1.0 \mathrm{eq}$.) was added as an internal standard. The vessel was wrapped with parafilm, placed into a violet light photoreaction setup and illuminated with stirring for 6 hours. Reaction progress was monitored using ${ }^{1} \mathrm{H}$ NMR analysis of reaction aliquots diluted with $\mathrm{CDCl}_{3}$. Crude product was purified using previously reported methods, yielding a white amorphous solid $(15.0 \mathrm{mg}, 76 \%$ isolated yield). Isolated product matched literature values. ${ }^{6}$

${ }^{1} \mathrm{H}$ NMR (600 MHz, $\left.\mathrm{CDCl}_{3}\right): \delta 8.37(\mathrm{~d}, \mathrm{~J}=8.0 \mathrm{~Hz}, 1 \mathrm{H}), 8.08(\mathrm{~d}, \mathrm{~J}=8.1 \mathrm{~Hz}, 1 \mathrm{H}), 8.02(\mathrm{~d}, \mathrm{~J}=8.0 \mathrm{~Hz}$, $1 \mathrm{H}), 7.80(\mathrm{t}, \mathrm{J}=7.8 \mathrm{~Hz}, 1 \mathrm{H}), 7.56(\mathrm{t}, \mathrm{J}=7.6 \mathrm{~Hz}, 1 \mathrm{H}), 7.56(\mathrm{t}, \mathrm{J}=7.6 \mathrm{~Hz}, 1 \mathrm{H}), 7.46(\mathrm{t}, \mathrm{J}=7.9 \mathrm{~Hz}, 1 \mathrm{H})$, $7.35-7.29(\mathrm{~m}, 2 \mathrm{H}) \mathrm{ppm}$.

${ }^{13} \mathrm{C}$ NMR $\left(151 \mathrm{MHz}, \mathrm{CDCl}_{3}\right): \delta 161.1,151.2,134.8,130.5,130.4,128.8,124.5,122.7,121.7$, $117.97,117.7 \mathrm{ppm}$.

Table S4: Percent yield by ${ }^{1} \mathrm{H}$ NMR for different photoreaction setups taken over the time course of benchmark 2.

\begin{tabular}{l|cccccccc|} 
& $\begin{array}{c}\mathbf{0} \\
\mathbf{m i n}\end{array}$ & $\begin{array}{c}\mathbf{1 5} \\
\mathbf{m i n}\end{array}$ & $\begin{array}{c}\mathbf{3 0} \\
\mathbf{m i n}\end{array}$ & $\begin{array}{c}\mathbf{4 5} \\
\mathbf{m i n}\end{array}$ & $\begin{array}{c}\mathbf{6 0} \\
\mathbf{m i n}\end{array}$ & $\begin{array}{c}\mathbf{1 2 0} \\
\mathbf{m i n}\end{array}$ & $\begin{array}{c}\mathbf{2 4 0} \\
\mathbf{m i n}\end{array}$ & $\begin{array}{c}\mathbf{3 6 0} \\
\mathbf{m i n}\end{array}$ \\
\cline { 2 - 9 } $\begin{array}{c}\text { WPP - 395 nm - } \\
\begin{array}{c}\text { Single Reaction } \\
\text { WPP - 395 nm - }\end{array}\end{array}$ & $0 \%$ & $9 \%$ & $27 \%$ & $48 \%$ & $60 \%$ & $69 \%$ & $72 \%$ & $75 \%$ \\
\cline { 2 - 9 } $\begin{array}{c}\text { Multiple Reaction } \\
400 \mathrm{~nm} \text { LED Strip } \\
\text { Photoreactor }\end{array}$ & $0 \%$ & $0 \%$ & $3 \%$ & $3 \%$ & $6 \%$ & $15 \%$ & $42 \%$ & $75 \%$ \\
\cline { 2 - 9 } & $0 \%$ & $0 \%$ & $0 \%$ & $0 \%$ & $0 \%$ & $0 \%$ & $0 \%$ & $1.5 \%$ \\
\hline
\end{tabular}




\subsection{Benchmark 3 - Os-photocatalyzed Trifluoromethylation of N-Methyl-2-pyridone}

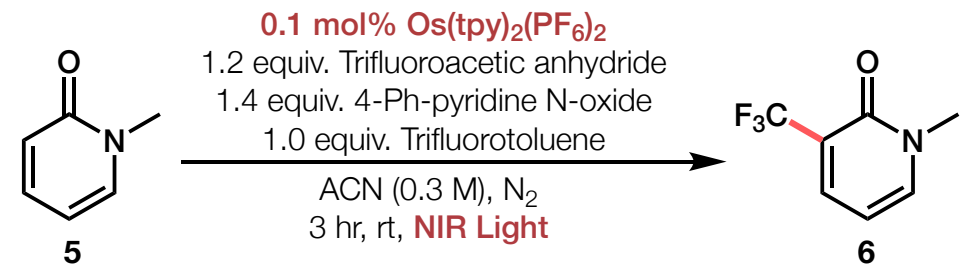

$0.31 \mathrm{mmol}$ scale

Procedure: To a $4 \mathrm{~mL}$ vial equipped with a stir bar were added $\mathrm{N}$-methyl-2-pyridone $(30.4 \mu \mathrm{L}$, $0.31 \mathrm{mmol}, 1.0$ eq.), 4-phenylpyridine $N$-oxide $(75.3 \mathrm{mg}, 0.44 \mathrm{mmol}, 1.4$ eq.) and ACN (1 mL, 0.3 M) under air. A concentrated stock solution of $\mathrm{Os}\left(\mathrm{tpy}_{2}\right)\left(\mathrm{PF}_{6}\right)_{2}$ in $\mathrm{ACN}(20 \mu \mathrm{L}, 0.00031 \mathrm{mmol}, 0.001$ eq.) was added, followed by trifluoroacetic anhydride (51.4 $\mu \mathrm{L}, 0.37 \mathrm{mmol}, 1.2 \mathrm{eq}$.). The resulting mixture was stirred for 5 minutes, the vessel was capped under air, and trifluorotoluene $(38.0 \mu \mathrm{L}$, $0.31 \mathrm{mmol}, 1.0$ eq.) was added as an internal standard. The reaction vessel was placed into a NIR light photoreaction setup and illuminated with stirring for 3 hours. Reaction progress was determined using ${ }^{19} \mathrm{~F}$ NMR analysis of reaction aliquots diluted with $\mathrm{CDCl}_{3}$. Crude Product was purified by flash column chromatography (1:1 ethyl acetate:n-pentane) followed by high performance liquid chromatography using a linear solvent gradient over 25 minutes at a flow rate of $12 \mathrm{~mL} / \mathrm{min}(10-80 \% \mathrm{~B}$, solvent $A=0.1 \%$ trifluoroacetic acid in filtered and degassed Millipore $\mathrm{H}_{2} \mathrm{O}$, solvent $\mathrm{B}=0.1 \%$ trifluoroacetic acid in $\mathrm{ACN}$ ). A tan amorphous solid was obtained in $44 \%$ isolated yield $(24.3 \mathrm{mg})$. Isolated product matched literature values. ${ }^{7}$

Os(tpy) $)_{2}\left(\mathrm{PF}_{6}\right)_{2}$ photocatalyst was synthesized according to reported methods ( $76 \%$ isolated yield, $244.8 \mathrm{mg}$, black amorphous solid) and matched literature values. ${ }^{3}$

${ }^{1} \mathrm{H}$ NMR $\left(600 \mathrm{MHz}, \mathrm{CDCl}_{3}\right): \delta 7.75(\mathrm{~d}, \mathrm{~J}=7.1 \mathrm{~Hz}, 1 \mathrm{H}), 7.51(\mathrm{~d}, \mathrm{~J}=6.7 \mathrm{~Hz}, 1 \mathrm{H}), 6.25-6.20(\mathrm{~m}$, $1 \mathrm{H}), 3.6(\mathrm{~s}, 3 \mathrm{H}) \mathrm{ppm}$.

${ }^{13} \mathrm{C}$ NMR (151 MHz, CDCl $): \delta 158.9,142.2,138.8$ (q, J $\left.\mathrm{J}_{\mathrm{C}-\mathrm{F}}=5 \mathrm{~Hz}\right) 122.7$ (q, J $\left.\mathrm{J}_{\mathrm{C}-\mathrm{F}}=271 \mathrm{~Hz}\right), 120.4$ $\left(\mathrm{q}, \mathrm{J}_{\mathrm{C}-\mathrm{F}}=31 \mathrm{~Hz}\right), 103.9,37.9 \mathrm{ppm}$.

${ }^{19} \mathrm{~F} \mathrm{NMR}\left(564 \mathrm{MHz}, \mathrm{CDCl}_{3}\right): \delta$-66.05 (3F) ppm.

Table S5: Percent yield by ${ }^{1} \mathrm{H}$ NMR for different photoreaction setups taken over the time course of benchmark 3.

\begin{tabular}{|c|c|c|c|c|c|c|c|c|c|c|c|}
\hline & $\begin{array}{c}0 \\
\min \end{array}$ & $\begin{array}{c}5 \\
\min \end{array}$ & $\begin{array}{c}10 \\
\min \end{array}$ & $\begin{array}{c}15 \\
\min \end{array}$ & $\begin{array}{c}20 \\
\min \end{array}$ & $\begin{array}{l}30 \\
\min \end{array}$ & $\begin{array}{c}45 \\
\min \end{array}$ & $\begin{array}{c}60 \\
\min \end{array}$ & $\begin{array}{l}90 \\
\min \end{array}$ & $\begin{array}{l}120 \\
\min \end{array}$ & $\begin{array}{l}180 \\
\min \end{array}$ \\
\hline $\begin{array}{l}\text { WPP - } 730 \mathrm{~nm} \text { - } \\
\text { Single Reaction }\end{array}$ & $0 \%$ & $8 \%$ & $19 \%$ & $28 \%$ & $36 \%$ & $44 \%$ & $48 \%$ & $49 \%$ & $49 \%$ & $49 \%$ & $49 \%$ \\
\hline $\begin{array}{l}\text { WPP - } 730 \mathrm{~nm} \text { - } \\
\text { Multiple Reaction }\end{array}$ & $0 \%$ & $3 \%$ & $7 \%$ & $12 \%$ & $17 \%$ & $25 \%$ & $35 \%$ & $41 \%$ & $46 \%$ & $48 \%$ & $48 \%$ \\
\hline $\begin{array}{c}630 \text { nm LED Strip } \\
\text { Photoreactor }\end{array}$ & $0 \%$ & $1 \%$ & $3 \%$ & $5 \%$ & $7 \%$ & $11 \%$ & $17 \%$ & $22 \%$ & $31 \%$ & $35 \%$ & $42 \%$ \\
\hline
\end{tabular}




\subsection{Benchmark 4 - Trifluoromethylation of 1,3,5-Trimethoxybenzene}

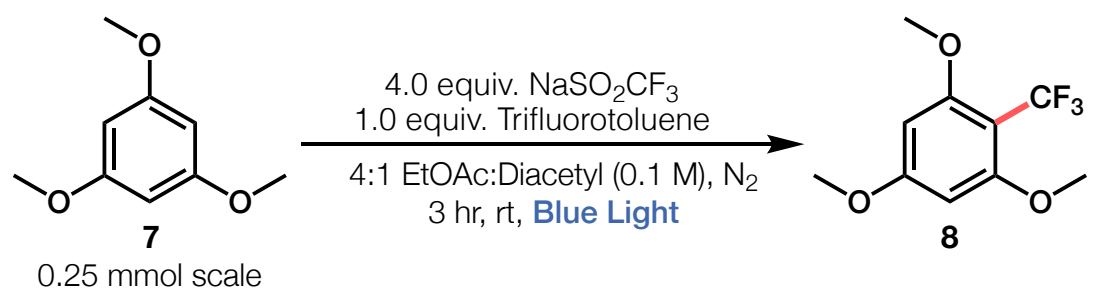

Procedure: To a $4 \mathrm{~mL}$ vial equipped with a stir bar were added sodium trifluoromethylsulfinate (156.1 mg, $1.0 \mathrm{mmol}, 4.0$ eq.) and 1,3,5-trimethoxybenzene $(42.1 \mathrm{mg}, 0.25 \mathrm{mmol}, 1.0$ eq.) followed by a $4: 1$ mixture of EtOAc:Diacetyl $(2.5 \mathrm{~mL}, 0.1 \mathrm{M})$ under air. The vessel was then capped and sparged with $\mathrm{N}_{2}$ for 15 minutes at $0{ }^{\circ} \mathrm{C}$. The reaction vessel was warmed to room temperature, and trifluorotoluene $(30.7 \mu \mathrm{L}, 0.25 \mathrm{mmol}, 1.0$ eq.) was added as an internal standard. The vessel was wrapped with parafilm, placed into a blue light photoreaction setup and illuminated with stirring for 3 hours. Reaction progress was monitored using ${ }^{19} \mathrm{~F}$ NMR analysis of reaction aliquots diluted with $\mathrm{CDCl}_{3}$. Crude product was purified using previously reported methods, yielding a white amorphous solid (43.9 $\mathrm{mg}, 74 \%$ isolated yield). Isolated product matched literature values. ${ }^{3}$

${ }^{1} \mathrm{H}$ NMR $\left(600 \mathrm{MHz}, \mathrm{CDCl}_{3}\right): \delta 6.13(\mathrm{~s}, 2 \mathrm{H}), 3.83(\mathrm{~s}, 9 \mathrm{H}) \mathrm{ppm}$.

${ }^{13} \mathrm{C}$ NMR $\left(151 \mathrm{MHz}, \mathrm{CDCl}_{3}\right): \delta$ 163.5, 160.41, 124.4 (q, JC-F $\left.=273 \mathrm{~Hz}\right), 100.3$ (q, JC-F $=30 \mathrm{~Hz}$ ), 91.2, 56.2, $55.4 \mathrm{ppm}$.

$\left.{ }^{19} \mathrm{~F} \mathrm{NMR} \mathrm{(376} \mathrm{MHz,} \mathrm{CDCl}_{3}\right): \delta-54.12(3 \mathrm{~F}) \mathrm{ppm}$.

Table S6: Percent yield by ${ }^{1} \mathrm{H}$ NMR for different photoreaction setups taken over the time course of benchmark 4 .

\begin{tabular}{|c|c|c|c|c|c|c|}
\hline & $\begin{array}{c}0 \\
\min \end{array}$ & $\begin{array}{c}30 \\
\text { min }\end{array}$ & $\begin{array}{c}60 \\
\text { min }\end{array}$ & $\begin{array}{c}90 \\
\text { min }\end{array}$ & $\begin{array}{l}120 \\
\text { min }\end{array}$ & $\begin{array}{l}180 \\
\text { min }\end{array}$ \\
\hline $\begin{array}{l}\text { WPP - } 450 \mathrm{~nm} \text { - } \\
\text { Sinale Reaction }\end{array}$ & $2 \%$ & $52 \%$ & $64 \%$ & $70 \%$ & $73 \%$ & $72 \%$ \\
\hline $\begin{array}{l}\text { WPP - } 450 \mathrm{~nm}- \\
\text { Multiple Reaction }\end{array}$ & $1 \%$ & $34 \%$ & $46 \%$ & $56 \%$ & $61 \%$ & $71 \%$ \\
\hline $\begin{array}{l}\text { Kessil A160WE } \\
\text { LED Lamp }\end{array}$ & $0 \%$ & $24 \%$ & $32 \%$ & $37 \%$ & $40 \%$ & $50 \%$ \\
\hline $\begin{array}{c}450 \text { nm LED Strip } \\
\text { Photoreactor }\end{array}$ & $0 \%$ & $13 \%$ & $22 \%$ & $28 \%$ & $31 \%$ & $36 \%$ \\
\hline CFL Bulb & $0 \%$ & $3 \%$ & $4 \%$ & $8 \%$ & $11 \%$ & $15 \%$ \\
\hline
\end{tabular}




\subsection{Benchmark 5 - Ru-photocatalyzed Trifluoromethylation of 2-Acetyl-N-boc-pyrrole}

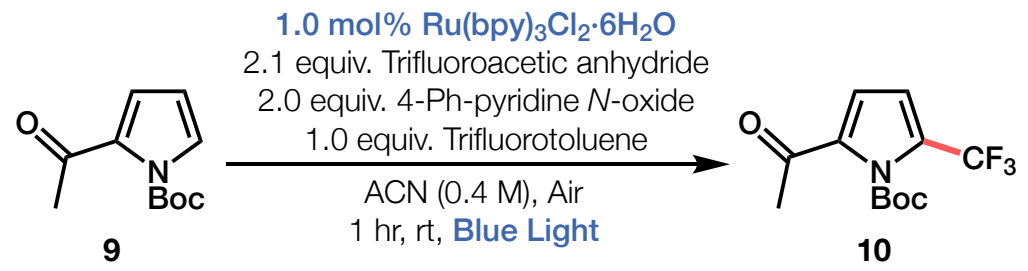

$0.8 \mathrm{mmol}$ scale

Procedure: To a $4 \mathrm{~mL}$ vial equipped with a stir bar were added $\mathrm{Ru}(\mathrm{bpy})_{3} \mathrm{Cl}_{2} \cdot 6 \mathrm{H} 2 \mathrm{O}(6.0 \mathrm{mg}, 0.008$ mmol, 0.01 eq.) and 4-phenylpyridine $N$-oxide (273.9 mg, $1.6 \mathrm{mmol}, 2.0$ eq.) under air. A solution of tert-butyl 2-acetyl-1H-pyrrole-1-carboxylate (167.0 mg, $0.8 \mathrm{mmol}, 1.0$ eq.) in ACN (2 mL, 0.4 $\mathrm{M})$ was then added, followed by trifluoroacetic anhydride $(237.0 \mu \mathrm{L}, 1.68 \mathrm{mmol}, 2.1 \mathrm{eq}$.). The resulting solution was stirred for 5 minutes, the vessel was capped under air, and trifluorotoluene (100.0 $\mu \mathrm{L}, 0.8 \mathrm{mmol}, 1.0$ eq.) was added as an internal standard. The reaction vessel was opened to atmosphere using a 22G needle, placed into a blue light photoreaction setup and illuminated with stirring for 1 hour. Reaction progress was monitored using ${ }^{19} \mathrm{~F}$ NMR analysis of reaction aliquots diluted with $\mathrm{CDCl}_{3}$. Crude product was purified using previously reported methods, yielding a clear oil $\left(93.2 \mathrm{mg}, 42 \%\right.$ isolated yield). Isolated product matched literature values. ${ }^{8}$

Starting material was synthesized according to reported methods ( $55 \%$ isolated yield, $1725 \mathrm{mg}$, clear oil) and matched literature values. ${ }^{9}$

${ }^{1} \mathrm{H}$ NMR $\left(600 \mathrm{MHz}, \mathrm{CDCl}_{3}\right): \delta 6.80$ (d, J =4.0 Hz, 1H), 6.60 (d, J = 4.0 Hz, 1H), 2.47 (s, $\left.3 \mathrm{H}\right) 1.61$ (s, 9H) ppm.

${ }^{13} \mathrm{C}$ NMR (151 MHz, CDCl $): \delta 187.9,147.9,135.3$ (q, J $\left.\mathrm{J}_{C-F}=1 \mathrm{~Hz}\right), 129.5,127.2,126.5$ (q, JC-F = $40 \mathrm{~Hz}$ ), 120.0 (q, J $\left.\mathrm{J}_{\mathrm{C}-\mathrm{F}}=269 \mathrm{~Hz}\right), 116.3,112.3$, (q, J $\left.\mathrm{J}_{\mathrm{C}-\mathrm{F}}=4 \mathrm{~Hz}\right), 86.9,27.1,26.7 \mathrm{ppm}$.

${ }^{19} \mathrm{~F} \mathrm{NMR}\left(564 \mathrm{MHz}, \mathrm{CDCl}_{3}\right): \delta-58.9(3 \mathrm{~F}) \mathrm{ppm}$.

Table S7: Percent yield by ${ }^{1} \mathrm{H}$ NMR for different photoreaction setups taken over the time course of benchmark 5 .

\begin{tabular}{|c|c|c|c|c|c|c|c|c|}
\hline & $\begin{array}{c}0 \\
\min \end{array}$ & $\begin{array}{c}1 \\
\min \end{array}$ & $\begin{array}{c}3 \\
\min \end{array}$ & $\begin{array}{c}5 \\
\min \end{array}$ & $\begin{array}{c}10 \\
\min \end{array}$ & $\begin{array}{l}25 \\
\min \end{array}$ & $\begin{array}{l}30 \\
\min \end{array}$ & $\begin{array}{c}60 \\
\min \end{array}$ \\
\hline $\begin{array}{l}\text { WPP - } 450 \mathrm{~nm} \mathrm{-} \\
\text { Sinqle Reaction }\end{array}$ & $1 \%$ & $24 \%$ & $57 \%$ & $65 \%$ & $65 \%$ & $64 \%$ & $65 \%$ & $65 \%$ \\
\hline $\begin{array}{l}\text { WPP - } 450 \mathrm{~nm}- \\
\text { Multiple Reaction }\end{array}$ & $1 \%$ & $12 \%$ & $36 \%$ & $53 \%$ & $66 \%$ & $65 \%$ & $65 \%$ & $64 \%$ \\
\hline $\begin{array}{l}\text { Kessil A160WE } \\
\text { LED Lamp }\end{array}$ & $0 \%$ & $4 \%$ & $10 \%$ & $16 \%$ & $30 \%$ & $43 \%$ & $61 \%$ & $64 \%$ \\
\hline $\begin{array}{l}450 \text { nm LED Strip } \\
\text { Photoreactor }\end{array}$ & $0 \%$ & $0 \%$ & $1 \%$ & $3 \%$ & $7 \%$ & $12 \%$ & $23 \%$ & $44 \%$ \\
\hline CFL Bulb & $0 \%$ & $0 \%$ & $1 \%$ & $2 \%$ & $4 \%$ & $7 \%$ & $13 \%$ & $31 \%$ \\
\hline
\end{tabular}




\subsection{Benchmark 6-C-N Cross-coupling of 4-Bromobenzotrifluoride and Morpholine via Metallophotoredox catalysis}

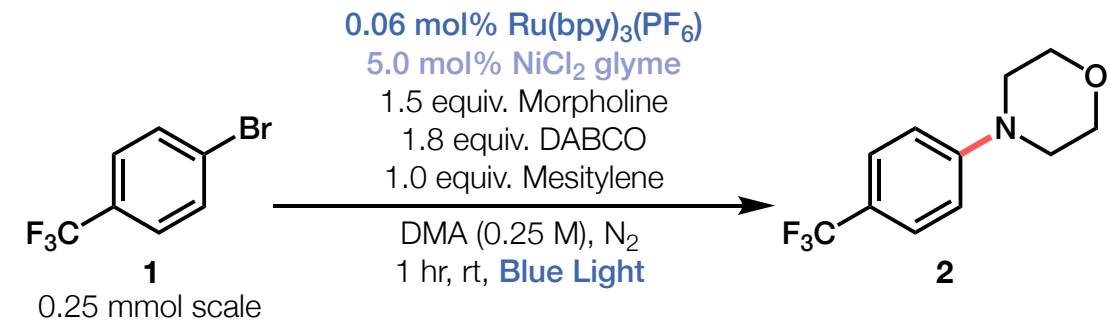

Procedure: To a $4 \mathrm{~mL}$ vial equipped with a stir bar were added $\mathrm{NiCl}_{2}$ glyme $(2.7 \mathrm{mg}, 0.0126$ mmol, 0.05 eq.), DABCO (50.5 mg, $0.45 \mathrm{mmol}, 1.8$ eq.) and dry DMA (1 mL, $0.25 \mathrm{M})$ under air. The vessel was capped and placed under $\mathrm{N}_{2}$. Morpholine ( $32.3 \mu \mathrm{L}, 0.375 \mathrm{mmol}, 1.5 \mathrm{eq}$.), 4bromobenzotrifluoride $(35.0 \mu \mathrm{L}, 0.25 \mathrm{mmol}, 1.0$ eq.) and a concentrated stock solution of $\mathrm{Ru}(\mathrm{bpy})_{3}\left(\mathrm{PF}_{6}\right)_{2}(20 \mu \mathrm{L}, 0.00015 \mathrm{mmol}, 0.0006$ equiv. $)$ in dry DMA were charged to the vessel. The resulting solution was sparged with $\mathrm{N}_{2}$ for 30 minutes at $0{ }^{\circ} \mathrm{C}$. The reaction vessel was warmed to room temperature, and mesitylene ( $34.8 \mu \mathrm{L}, 0.25 \mathrm{mmol}, 1.0$ eq.) was added as an internal standard. The vessel cap was wrapped with parafilm, placed into a blue light photoreaction setup and illuminated with stirring for 1 hour. Reaction progress was monitored using ${ }^{1} \mathrm{H}$ NMR analysis of reaction aliquots diluted with DMSO- $\mathrm{d}_{6}$. Crude product was purified using previously reported methods, yielding a white amorphous solid ( $94 \%$ isolated yield, $54.6 \mathrm{mg})$. Isolated product matched literature values. ${ }^{10}$

${ }^{1} \mathrm{H} \mathrm{NMR}\left(600 \mathrm{MHz}, \mathrm{CDCl}_{3}\right): \delta 7.50(\mathrm{~d}, \mathrm{~J}=8.7 \mathrm{~Hz}, 2 \mathrm{H}), 6.92(\mathrm{~d}, \mathrm{~J}=8.5 \mathrm{~Hz}, 2 \mathrm{H}), 3.86(\mathrm{t}, \mathrm{J}=5.0 \mathrm{~Hz}$, $4 \mathrm{H}) 3.23(\mathrm{t}, \mathrm{J}=5.0 \mathrm{~Hz}, 4 \mathrm{H}) \mathrm{ppm}$.

${ }^{13} \mathrm{C}$ NMR (151 MHz, CDCl $)$ ): $\delta 153.5,127.5,126.6\left(\mathrm{q}, \mathrm{J}_{\mathrm{C}-\mathrm{F}}=4 \mathrm{~Hz}\right), 124.7$ (q, $\left.\mathrm{J}_{\mathrm{C}-\mathrm{F}}=270 \mathrm{~Hz}\right), 121.1$ (q, J $\mathrm{J}_{\mathrm{C}-\mathrm{F}}=33 \mathrm{~Hz}$ ), 114.4, 66.8, $48.3 \mathrm{ppm}$.

${ }^{19} \mathrm{~F} \mathrm{NMR}\left(376 \mathrm{MHz}, \mathrm{CDCl}_{3}\right): \delta-61.4(3 \mathrm{~F}) \mathrm{ppm}$.

Table S8: Percent yields determined by ${ }^{1} \mathrm{H}$ NMR for different photoreaction setups over the course of benchmark 6 .

\begin{tabular}{|c|c|c|c|c|c|c|c|c|}
\hline & $\begin{array}{c}0 \\
\min \end{array}$ & $\begin{array}{c}2 \\
\min \end{array}$ & $\begin{array}{c}5 \\
\min \end{array}$ & $\begin{array}{c}10 \\
\min \end{array}$ & $\begin{array}{c}15 \\
\min \end{array}$ & $\begin{array}{c}20 \\
\min \end{array}$ & $\begin{array}{c}30 \\
\min \end{array}$ & $\begin{array}{l}60 \\
\min \end{array}$ \\
\hline $\begin{array}{l}\text { WPP - } 450 \mathrm{~nm}- \\
\text { Single Reaction* }\end{array}$ & $1.5 \%$ & $21 \%$ & $52.5 \%$ & $82.5 \%$ & $94.5 \%$ & $96 \%$ & $96 \%$ & $96 \%$ \\
\hline $\begin{array}{l}\text { WPP - } 450 \mathrm{~nm} \text { - } \\
\text { Multiple Reaction }\end{array}$ & $3 \%$ & $28.5 \%$ & $58.5 \%$ & $81 \%$ & $88.5 \%$ & $93 \%$ & $96 \%$ & $97.5 \%$ \\
\hline $\begin{array}{l}\text { Kessil A160WE } \\
\text { LED Lamp }\end{array}$ & $1.5 \%$ & $10.5 \%$ & $24 \%$ & $43.5 \%$ & $57 \%$ & $66 \%$ & $82.5 \%$ & $93 \%$ \\
\hline $\begin{array}{l}450 \text { nm LED Strip } \\
\text { Photoreactor }\end{array}$ & $3 \%$ & $6 \%$ & $13.5 \%$ & $22.5 \%$ & $31.5 \%$ & $39 \%$ & $49.5 \%$ & $70.5 \%$ \\
\hline CFL Bulb & $3 \%$ & $4.5 \%$ & $6 \%$ & $9 \%$ & $12 \%$ & $15 \%$ & $19.5 \%$ & $34.5 \%$ \\
\hline
\end{tabular}




\subsection{Reaction Monitoring by NMR}

Aliquot procedure: Using a narrow gauge $50 \mu \mathrm{L}$ Hamilton syringe, the septum of the reaction vessel was pierced and $20 \mu \mathrm{L}$ of reaction solution was withdrawn while the vessel remained in the photoreaction setup. The syringe was removed, and the puncture hole was sealed with parafilm. The aliquot was delivered to a test tube, diluted with $600 \mu \mathrm{L}$ of deuterated solvent, transferred to an NMR tube and then held in a dark freezer until measurement by NMR spectroscopy. This procedure was repeated for each time point over the course of a time study.

Yield determination: Percent yield was determined by NMR analysis of reaction aliquots. Two well-resolved signals corresponding to the product and internal standard were integrated. Each integral was corrected for the number of nuclei represented by each corresponding signal. The corrected product integral was then divided by the corrected internal standard integral and multiplied by 100 to provide percent yield.

$$
\text { Percent Yield }=\frac{\left(I_{\text {Product }} / N_{\text {Product }}\right)}{\left(I_{\text {Intenral standard }} / N_{\text {Internal Standard }}\right)} * 100 \%
$$

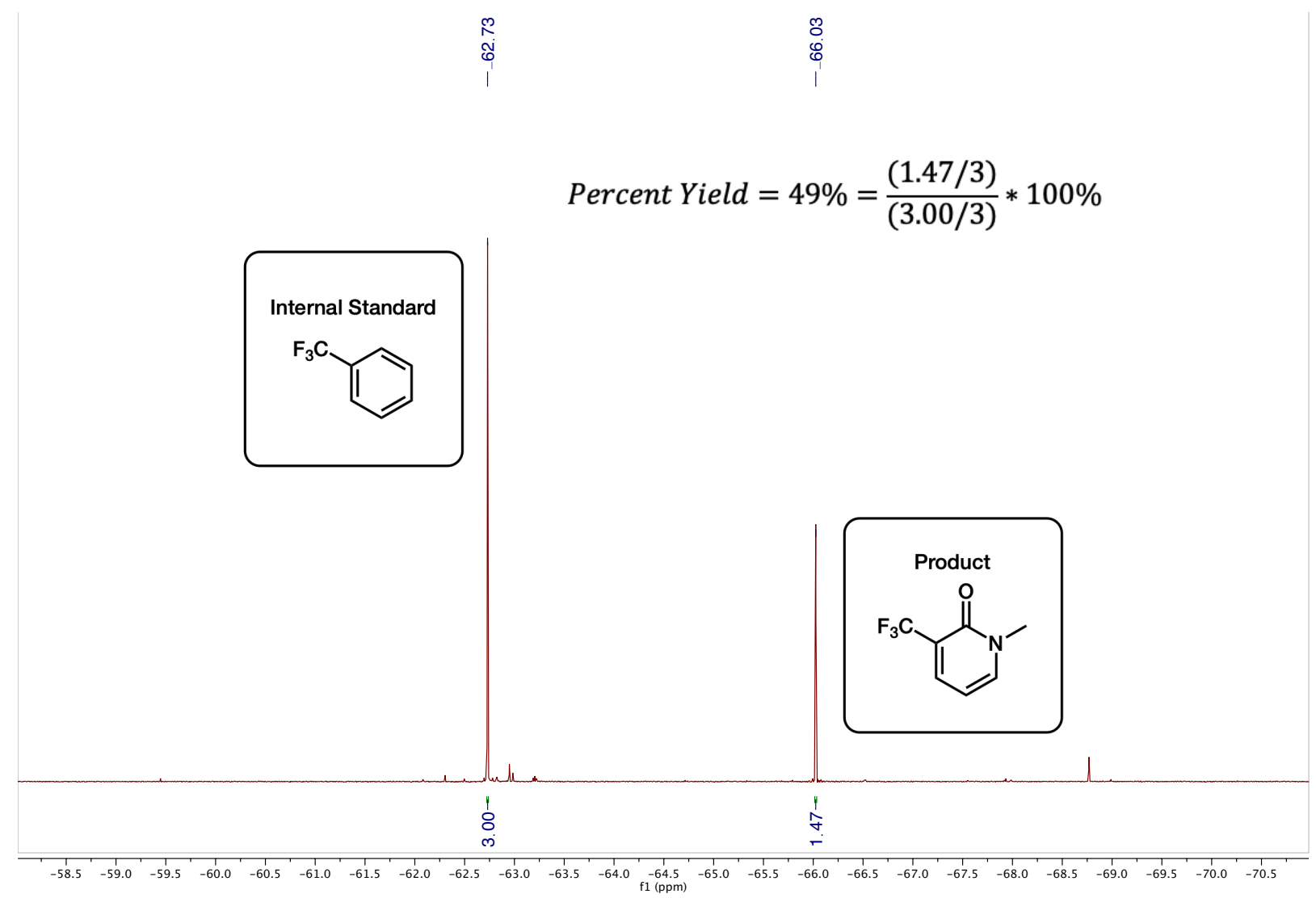

Figure S21: Example percent yield determination via ${ }^{19} \mathrm{~F}$ NMR analysis of an aliquot taken from benchmark 3. 


\section{Additional Experiments and Information}

\subsection{Position Equivalency Experiment}

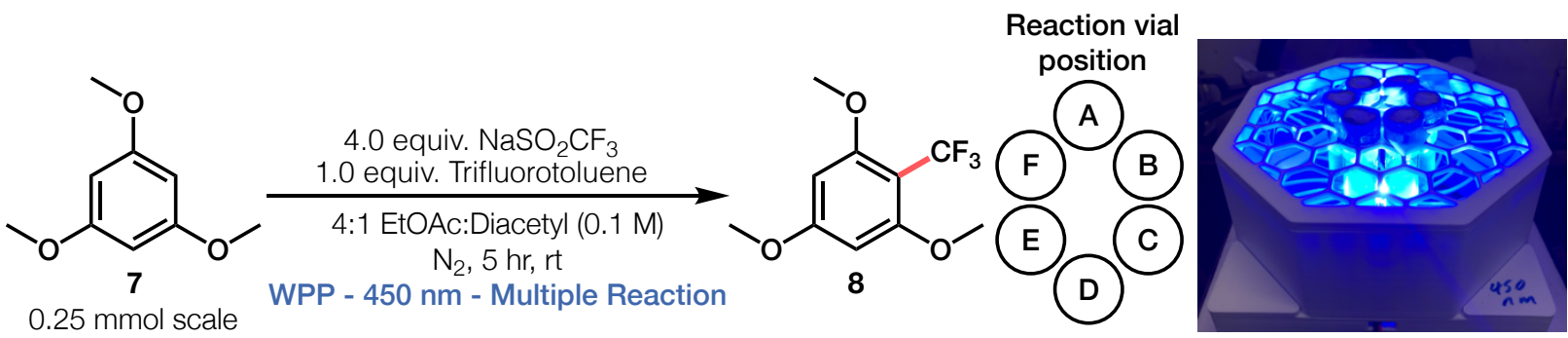

To evaluate if each position in the multiple reaction configuration of a WPP device fitted with a 4-mL reaction module provided equivalent performance, six simultaneous benchmark 4 reactions were conducted using the procedure outlined on page S22. At each time point, the reaction timer was paused, the WPP device was powered off to halt all photoreactions and aliquots were taken. Once all aliquots were taken, the timer was resumed and the WPP device was turned on to continue the time course experiment.

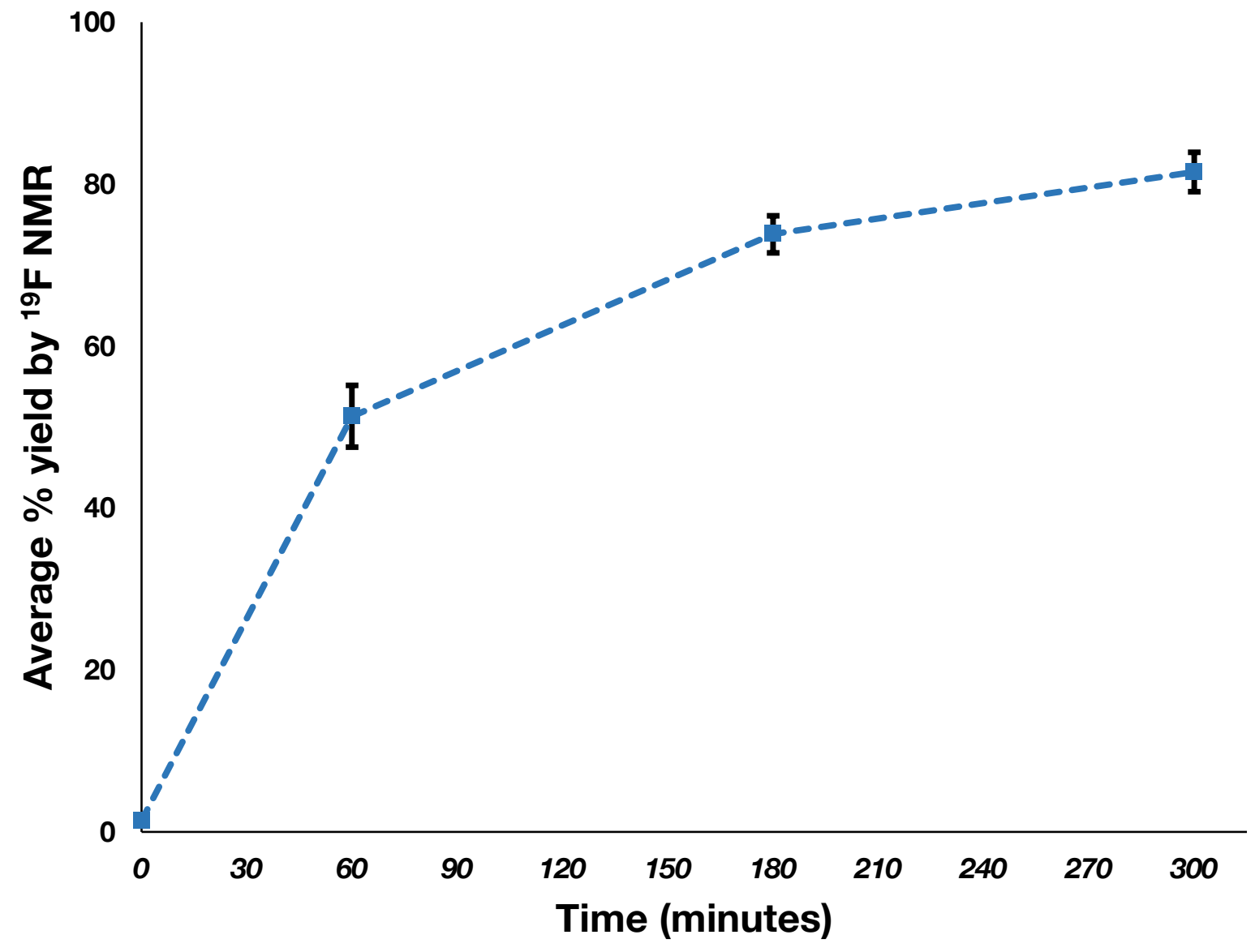

Figure S22: Average percent yield determined by ${ }^{19} \mathrm{~F}$ NMR of 6 simultaneous trials of benchmark 4 with error bars depicting standard deviation among all 6 trials. 
Table S9: Percent yields determined by ${ }^{19} \mathrm{~F}$ NMR for different vial positions over the course of the position equivalency experiment.

\begin{tabular}{|c|c|c|c|c|}
\hline & $\begin{array}{c}0 \\
\text { min }\end{array}$ & $\begin{array}{c}60 \\
\text { min }\end{array}$ & $\begin{array}{l}180 \\
\text { min }\end{array}$ & $\begin{array}{l}300 \\
\text { min }\end{array}$ \\
\hline Position A & $2 \%$ & $50 \%$ & $76 \%$ & $82 \%$ \\
\hline Position B & $1 \%$ & $46 \%$ & $72 \%$ & $82 \%$ \\
\hline Position C & $1 \%$ & $55 \%$ & $76 \%$ & $84 \%$ \\
\hline Position D & $2 \%$ & $57 \%$ & $75 \%$ & $84 \%$ \\
\hline Position E & $1 \%$ & $48 \%$ & $70 \%$ & $77 \%$ \\
\hline Position F & $1 \%$ & $52 \%$ & $72 \%$ & $80 \%$ \\
\hline Average & $1.4 \%$ & $51.3 \%$ & $73.8 \%$ & $81.5 \%$ \\
\hline Standard Deviation & $0.5 \%$ & $3.8 \%$ & $2.3 \%$ & $2.4 \%$ \\
\hline
\end{tabular}

\subsection{Scale-up Experiment}

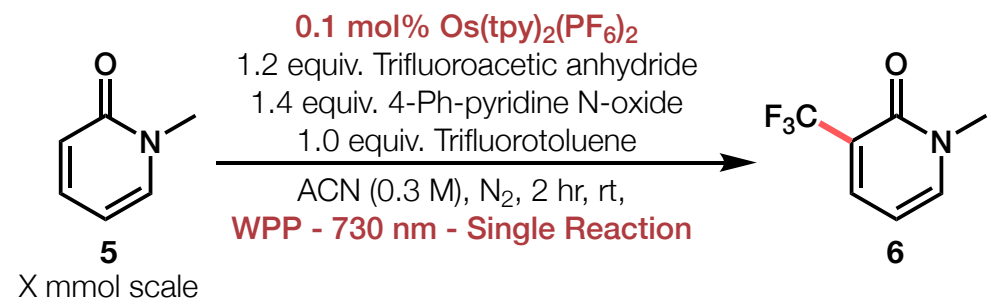

To evaluate WPP performance across common laboratory reaction scales, four benchmark 3 reactions were conducted using WPP devices fitted with 2-, 4-, 8- and 24-mL reaction modules in the single reaction configuration. Reactions were conducted at $0.1,0.31,1.0$ and $2.0 \mathrm{mmol}$ scale. Each reaction was carried out using the general procedure outlined on page S21. For the $1.0 \mathrm{mmol}$ trial, a tan amorphous solid was obtained in $39 \%$ isolated yield $(69.1 \mathrm{mg})$.

Table S10: Percent yields determined by ${ }^{19} \mathrm{~F}$ NMR for different reaction scales over the course of the scale-up experiment.

$0.1 \mathrm{mmol}$ (2-mL Vial)

$0.31 \mathrm{mmol}$ (4-mL vial)

$1.0 \mathrm{mmol}$ (8-mL Vial)

2.0 mmol (24-mL vial)

\begin{tabular}{|cc|}
\multicolumn{1}{c}{$\begin{array}{c}\text { min } \\
0 \%\end{array}$} & $\begin{array}{c}\mathbf{1 2 0} \\
\text { min }\end{array}$ \\
\hline $0 \%$ & $48 \%$ \\
\hline $0 \%$ & $49 \%$ \\
\hline $0 \%$ & $51 \%$ \\
\hline
\end{tabular}




\subsection{Cooling Efficiency With and Without Light Shield Module}

To evaluate WPP device cooling efficiency with and without the light shield module provided in the project repository, a $4 \mathrm{~mL}$ vial was equipped with a stir bar, charged with $2 \mathrm{~mL}$ of roomtemperature water and placed into the single reaction configuration of a WPP device fitted with a $450 \mathrm{~nm}$ LED star and 4-mL reaction module. A thermocouple was inserted midway into the vessel and allowed to rest against the vial wall. The WPP device was then covered with a light shield module printed in black PLA or left uncovered, switched on and vial temperature was monitored for one hour.

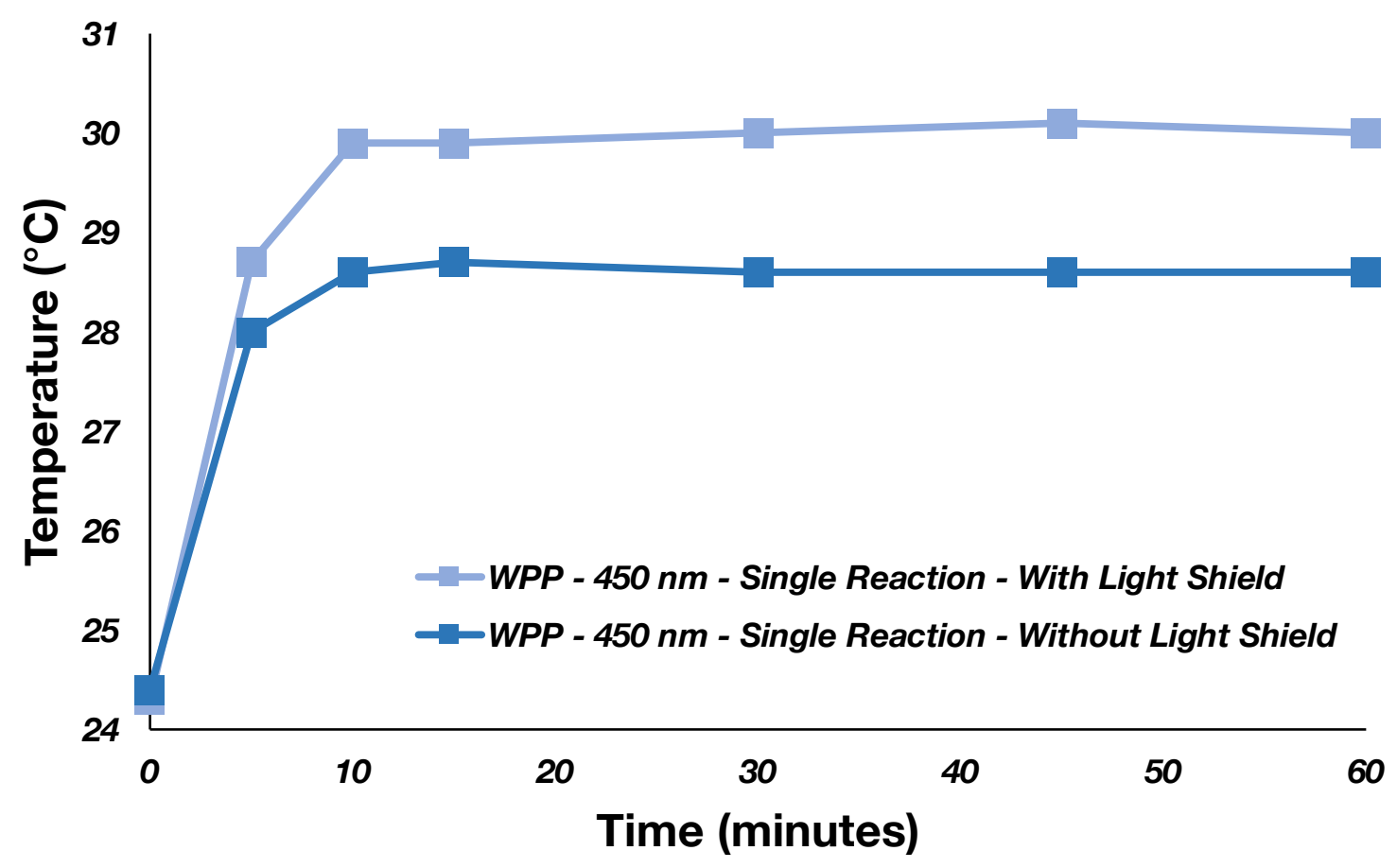

Figure S23: Temperatures of a vial in the single reaction configuration of a $450 \mathrm{~nm}$ WPP device with and without a light shield cover module.

Table S11: Measured temperatures of a vial in the single reaction configuration of a $450 \mathrm{~nm}$ WPP device with and without a light shield cover module over the course of one hour.

\begin{tabular}{|c|c|c|c|c|c|c|c|}
\hline & $\begin{array}{c}0 \\
\min \end{array}$ & $\begin{array}{c}5 \\
\min \end{array}$ & $\begin{array}{c}10 \\
\min \end{array}$ & $\begin{array}{c}15 \\
\min \end{array}$ & $\begin{array}{l}30 \\
\min \end{array}$ & $\begin{array}{c}45 \\
\min \end{array}$ & $\begin{array}{c}60 \\
\text { min }\end{array}$ \\
\hline 450 nm WPP device & $24.3^{\circ} \mathrm{C}$ & $28.7^{\circ} \mathrm{C}$ & $29.9^{\circ} \mathrm{C}$ & $29.9^{\circ} \mathrm{C}$ & $30.0^{\circ} \mathrm{C}$ & $30.1^{\circ} \mathrm{C}$ & $30.0^{\circ} \mathrm{C}$ \\
\hline $\begin{array}{l}50 \mathrm{~nm} \text { WPP device } \\
\text { ithout light shield }\end{array}$ & $24.4^{\circ} \mathrm{C}$ & $28.0^{\circ} \mathrm{C}$ & $28.6^{\circ} \mathrm{C}$ & $28.7^{\circ} \mathrm{C}$ & $28.6^{\circ} \mathrm{C}$ & $28.6^{\circ} \mathrm{C}$ & $28.6^{\circ} \mathrm{C}$ \\
\hline
\end{tabular}




\subsection{Comparison to MacMillan Photoreactor}

A comparison of reactions conducted using a $450 \mathrm{~nm}$ WPP device and the commercial photoreactor described by MacMillan et al. in reference 4 is provided below. Reaction profiles for transformations conducted with the MacMillan photoreactor were taken from reference 4.

\section{Comparison of Benchmark 4 - Trifluoromethylation of 1,3,5-Trimethoxybenzene}

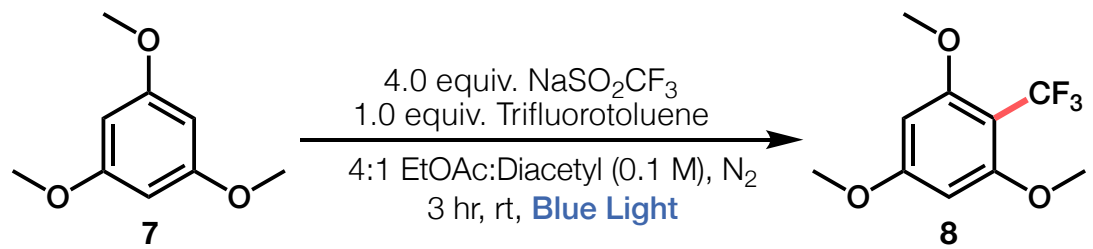

$0.25 \mathrm{mmol}$ scale

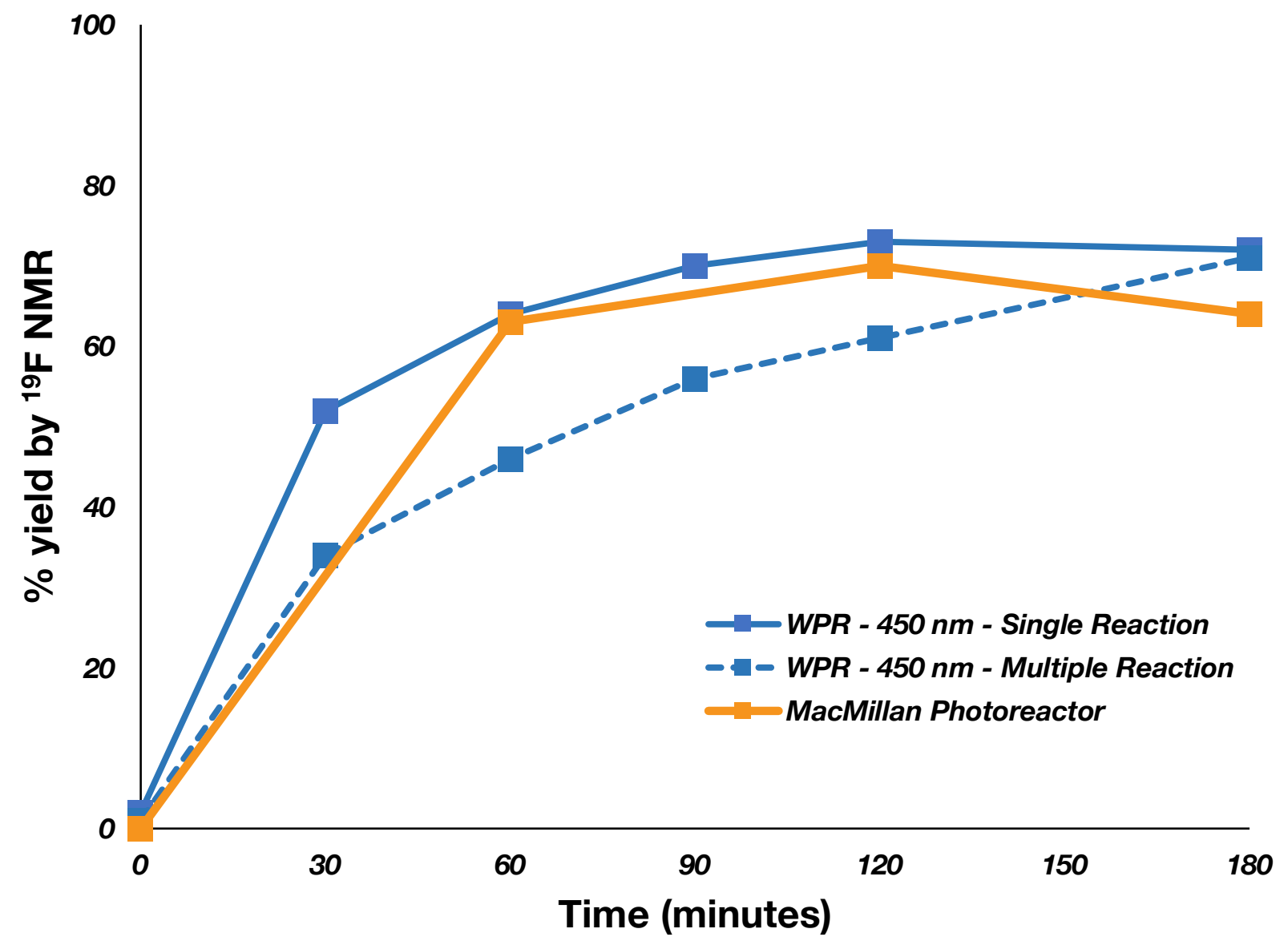

Figure S24: Comparison of percent yield determined by ${ }^{19} \mathrm{~F}$ NMR of benchmark 4 conducted with the MacMillan photoreactor and WPP device in single and multiple reaction configurations. 


\section{Comparison of Benchmark 5 - Ru-photocatalyzed \\ Trifluoromethylation of 2-Acetyl-N-boc-pyrrole}

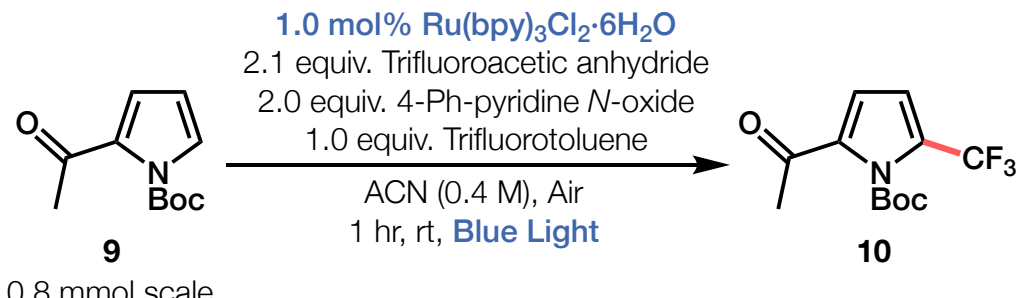

$0.8 \mathrm{mmol}$ scale

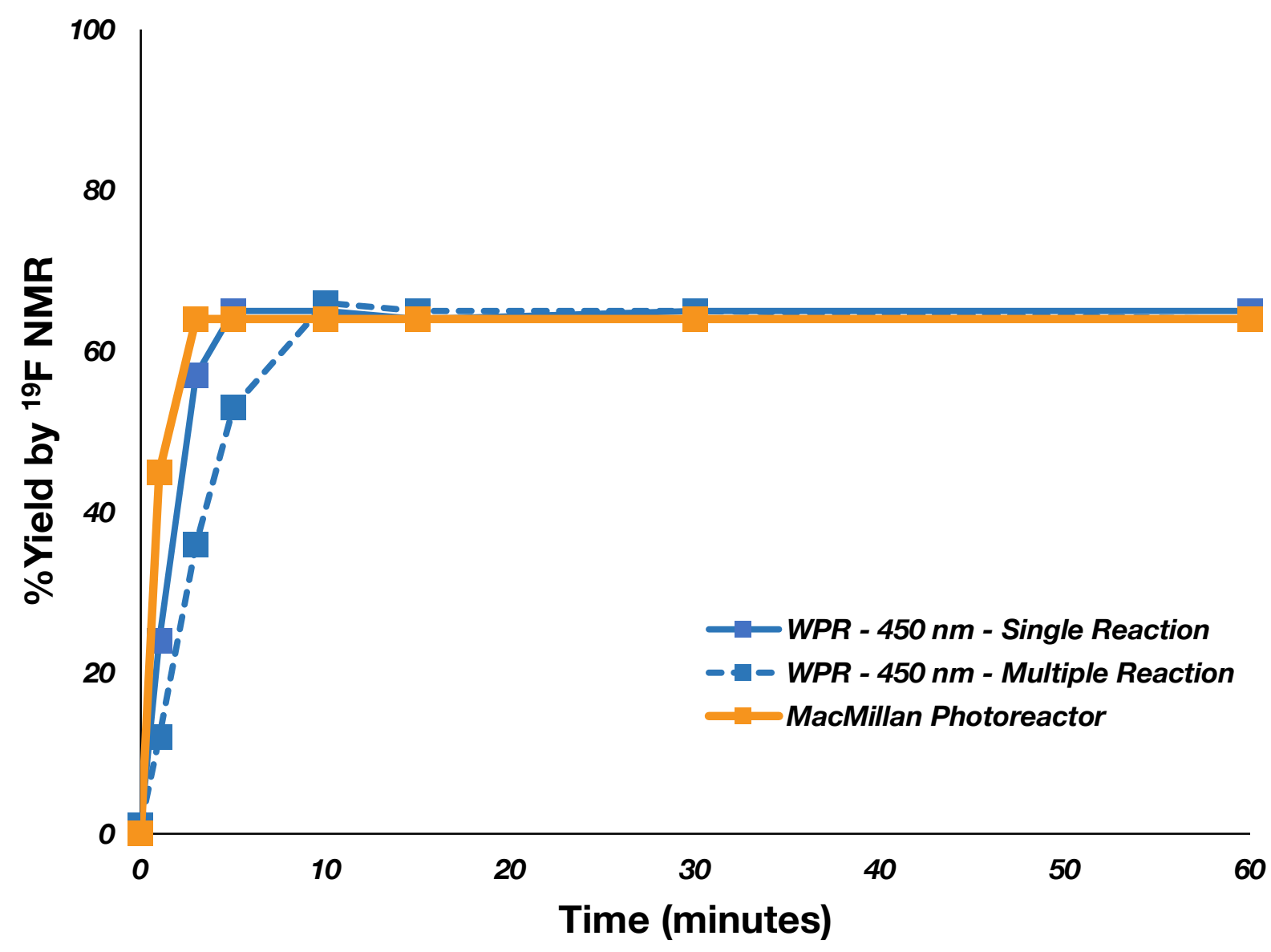

Figure S25: Comparison of percent yield determined by ${ }^{19} \mathrm{~F}$ NMR of benchmark 5 conducted with the MacMillan photoreactor and WPP device in single and multiple reaction configurations. 
Comparison of Benchmark 6 - C-N Cross-coupling of 4-Bromobenzotrifluoride and Morpholine via Metallophotoredox catalysis
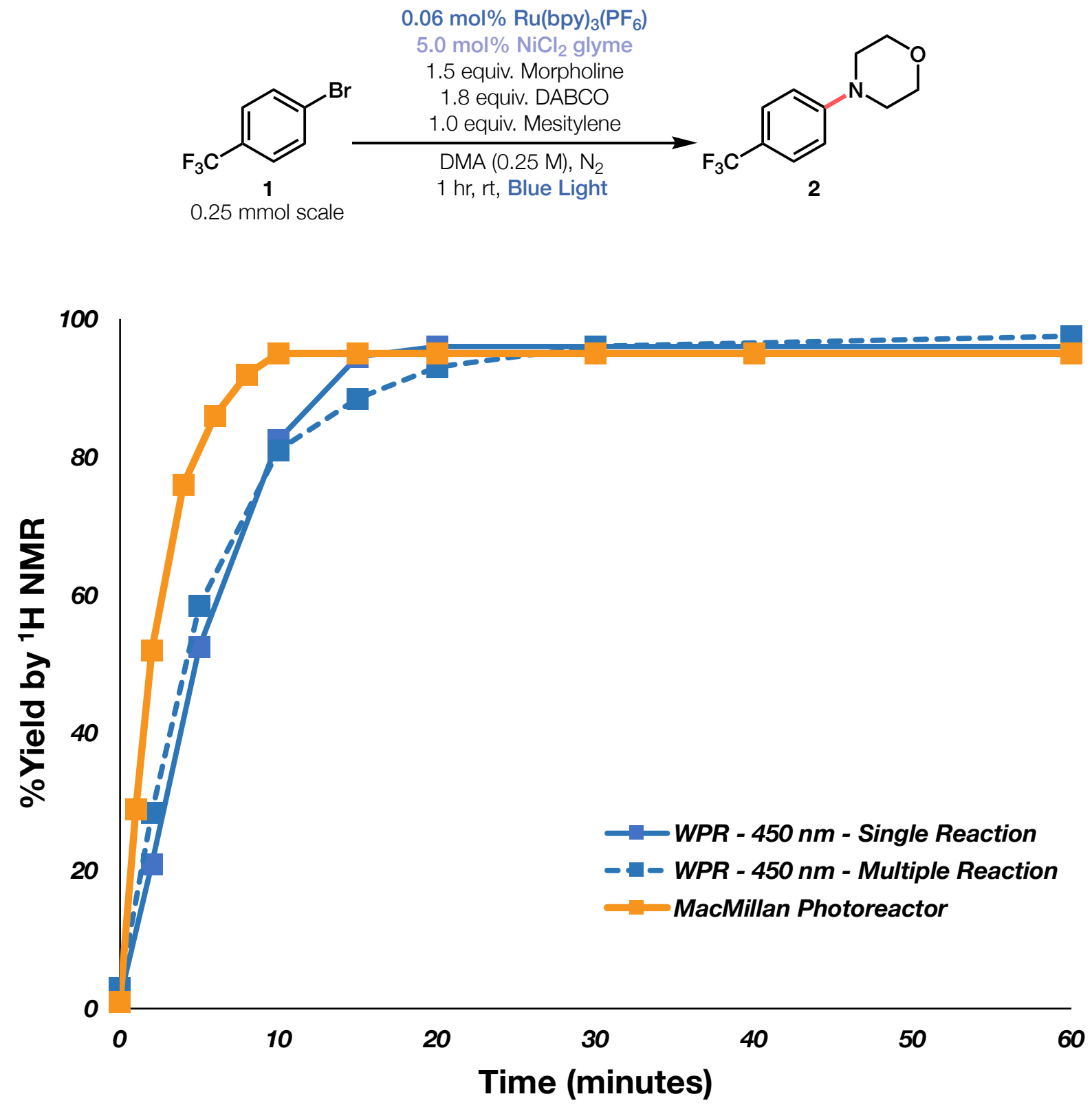

Figure S26: Comparison of percent yield determined by ${ }^{1} \mathrm{H}$ NMR of benchmark 6 conducted with the MacMillan photoreactor and WPP device in single and multiple reaction configurations. 


\section{Product Spectral Data}

\section{4-(4-(trifluoromethyl)phenyl)morpholine (2)}

${ }^{1} \mathrm{H} \mathrm{NMR}\left(400 \mathrm{MHz}, \mathrm{CDCl}_{3}\right)$

攴名

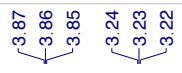
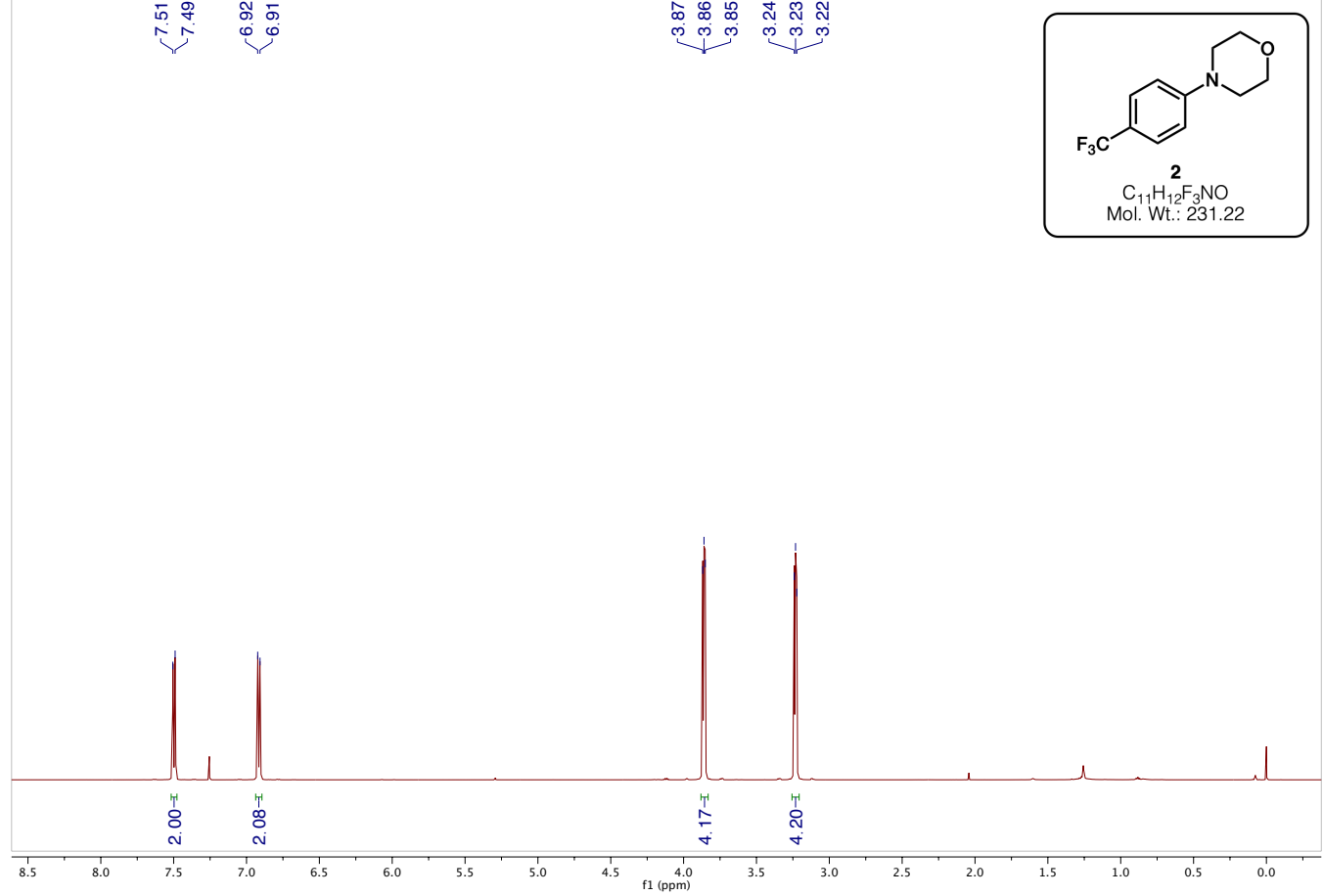

${ }^{13} \mathrm{C}$ NMR $\left(151 \mathrm{MHz}, \mathrm{CDCl}_{3}\right)$

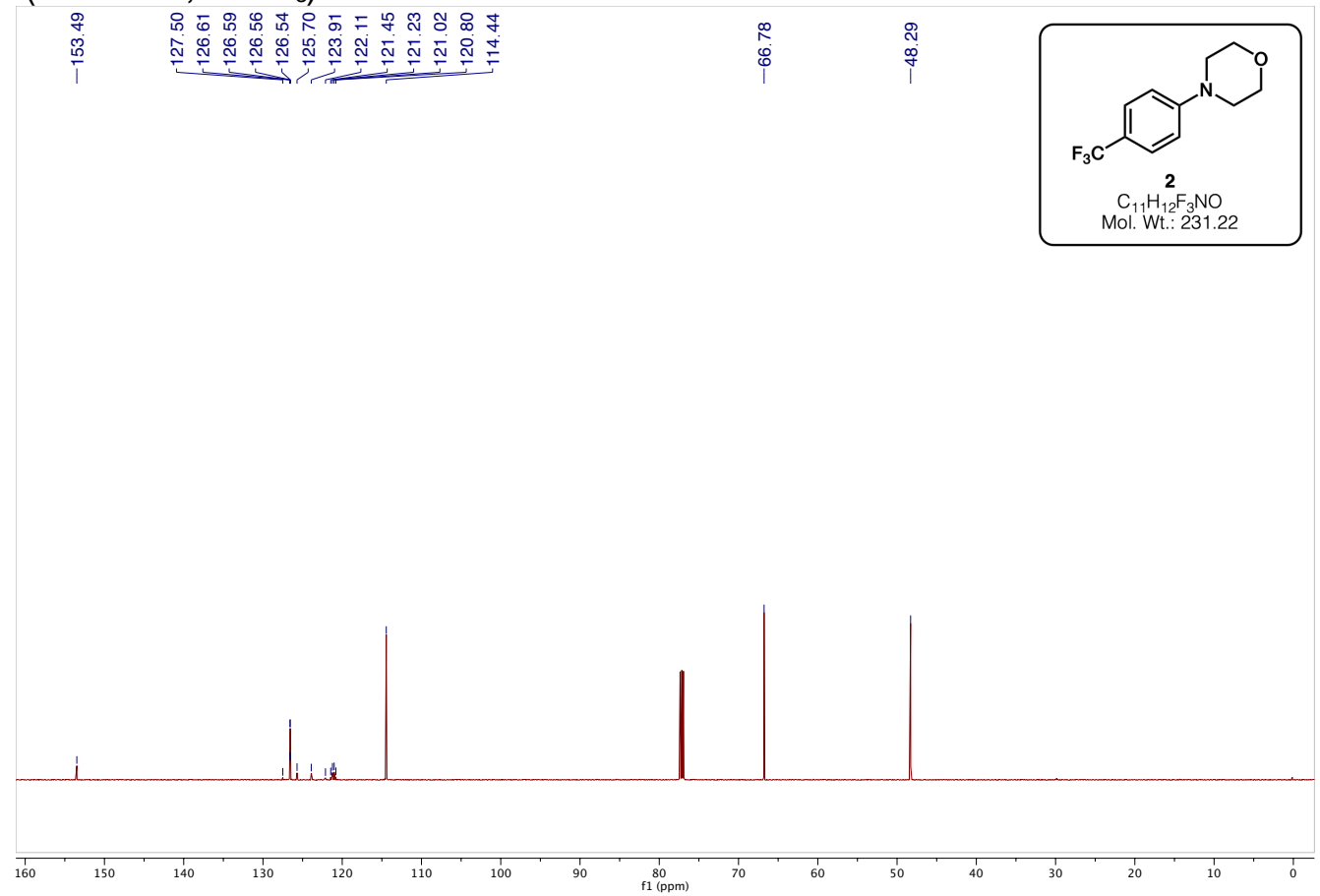


$\left.{ }^{19} \mathrm{~F} \mathrm{NMR} \mathrm{(376} \mathrm{MHz,} \mathrm{CDCl}_{3}\right)$
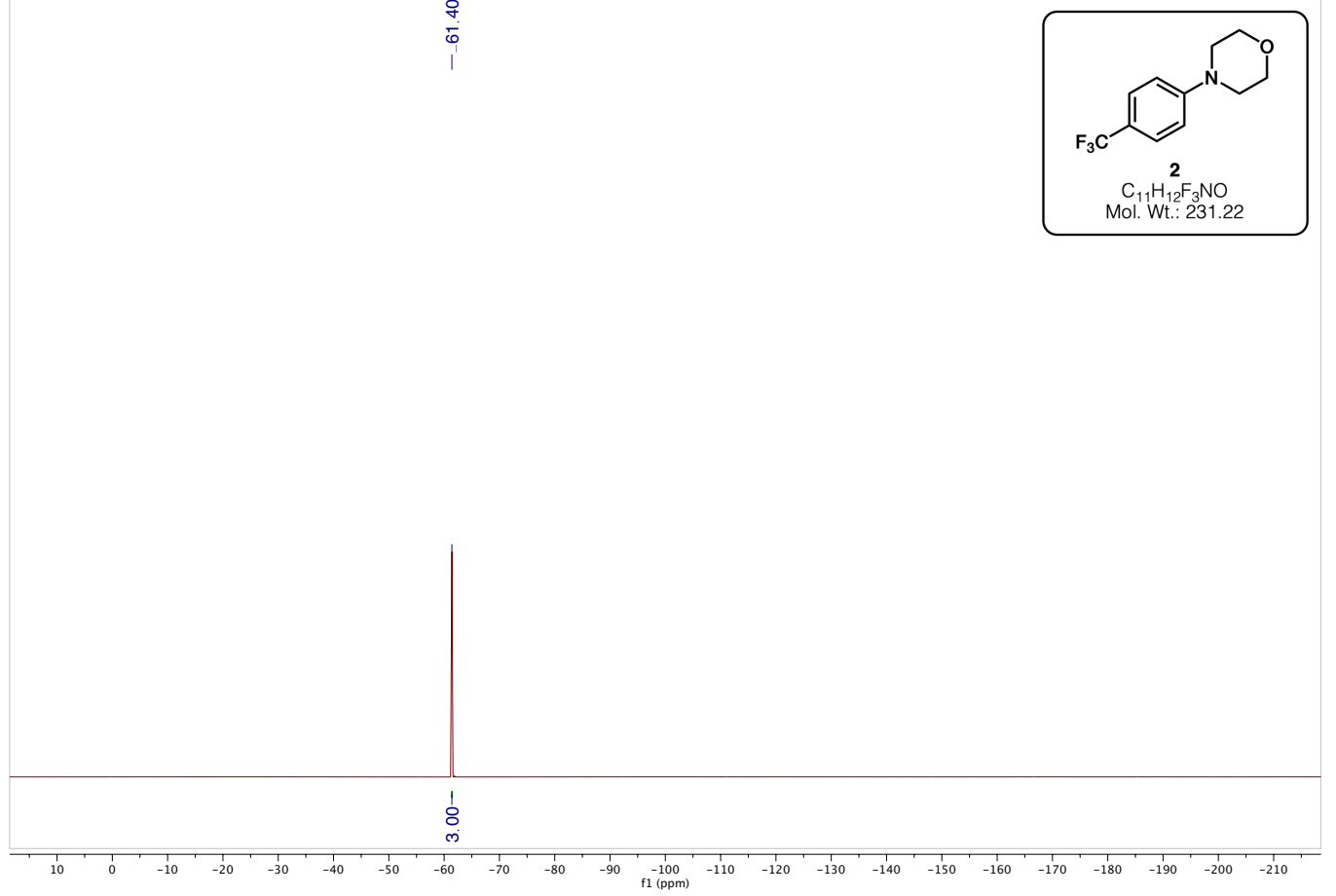

\section{H-benzo[c]chromen-6-one (4)}

${ }^{1} \mathrm{H}$ NMR $\left(600 \mathrm{MHz}, \mathrm{CDCl}_{3}\right)$

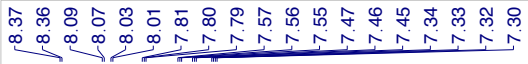

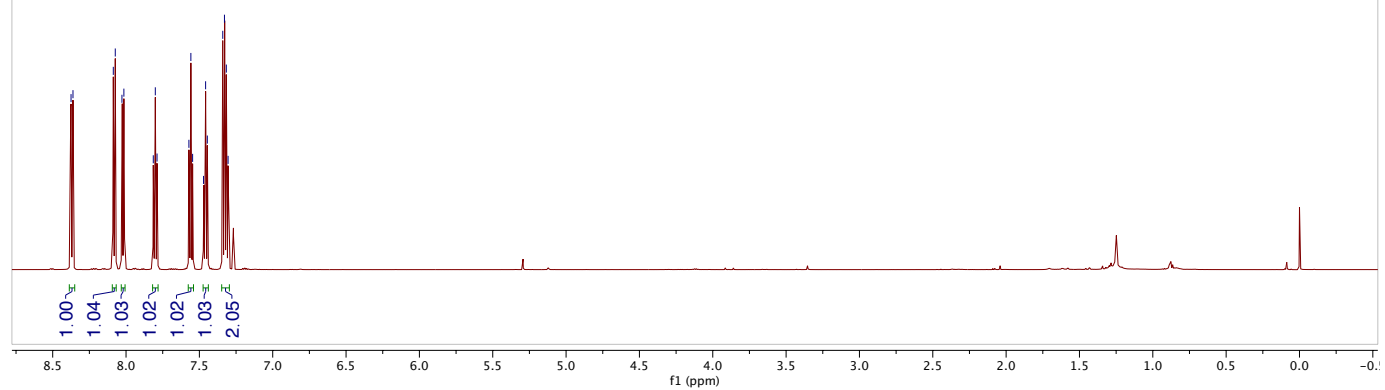


${ }^{13} \mathrm{C}$ NMR $\left(151 \mathrm{MHz}, \mathrm{CDCl}_{3}\right)$

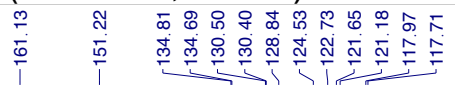
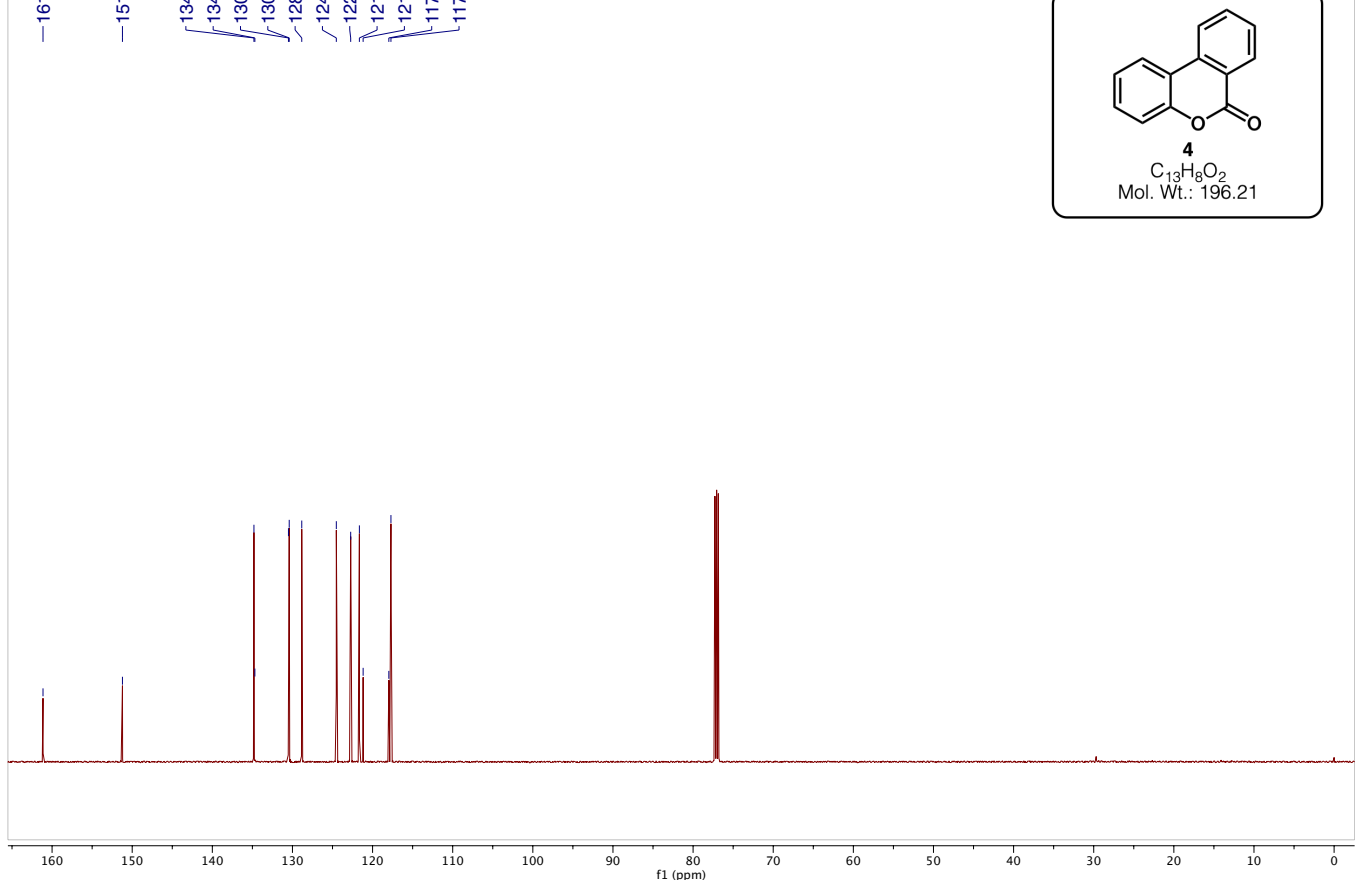

\section{1-methyl-3-(trifluoromethyl)-2(1H)-pyridone (6)}

${ }^{1} \mathrm{H}$ NMR $\left(600 \mathrm{MHz}, \mathrm{CDCl}_{3}\right)$

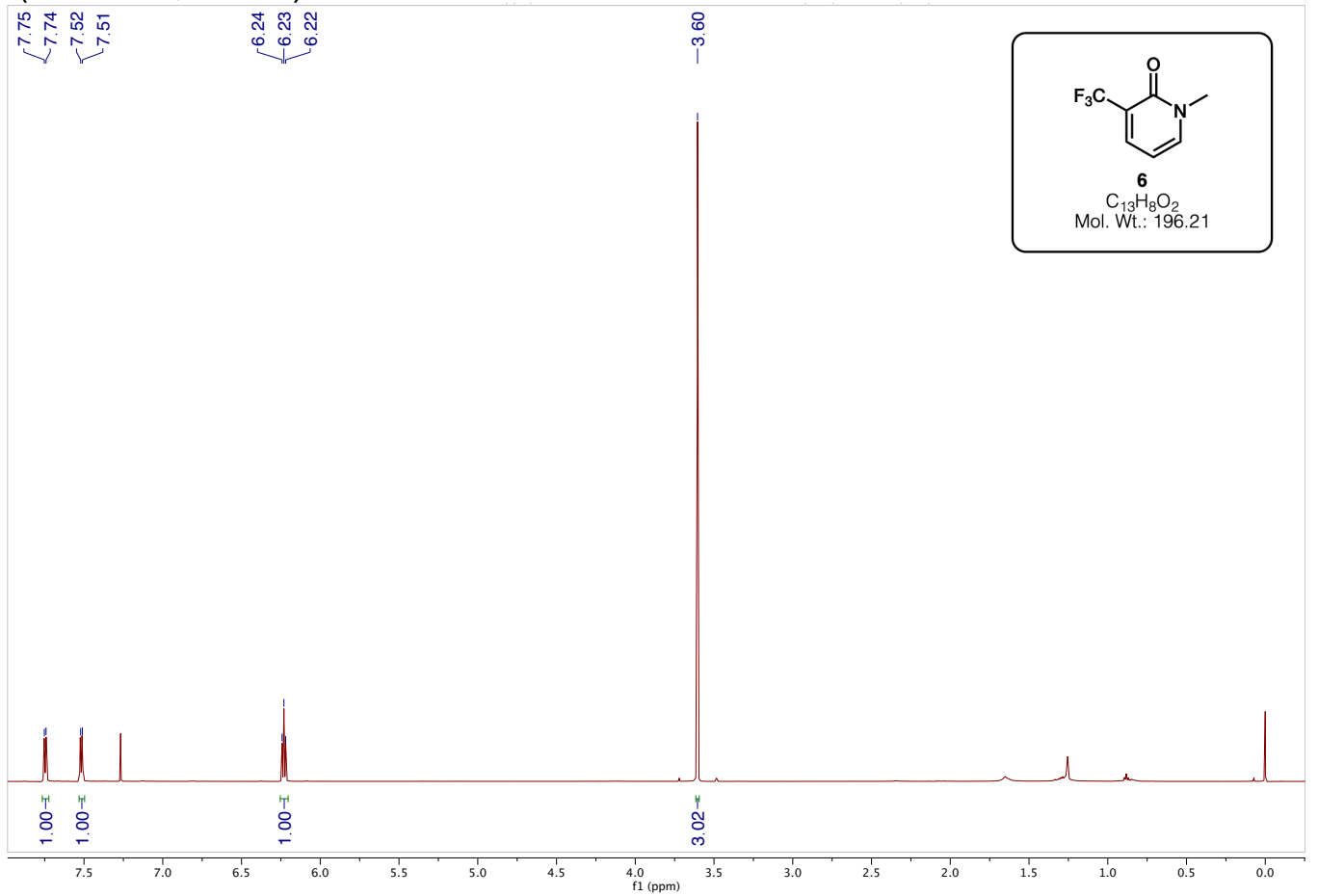


${ }^{13} \mathrm{C}$ NMR (151 MHz, $\mathrm{CDCl}_{3}$ )

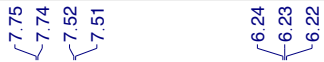

$\stackrel{8}{9}$

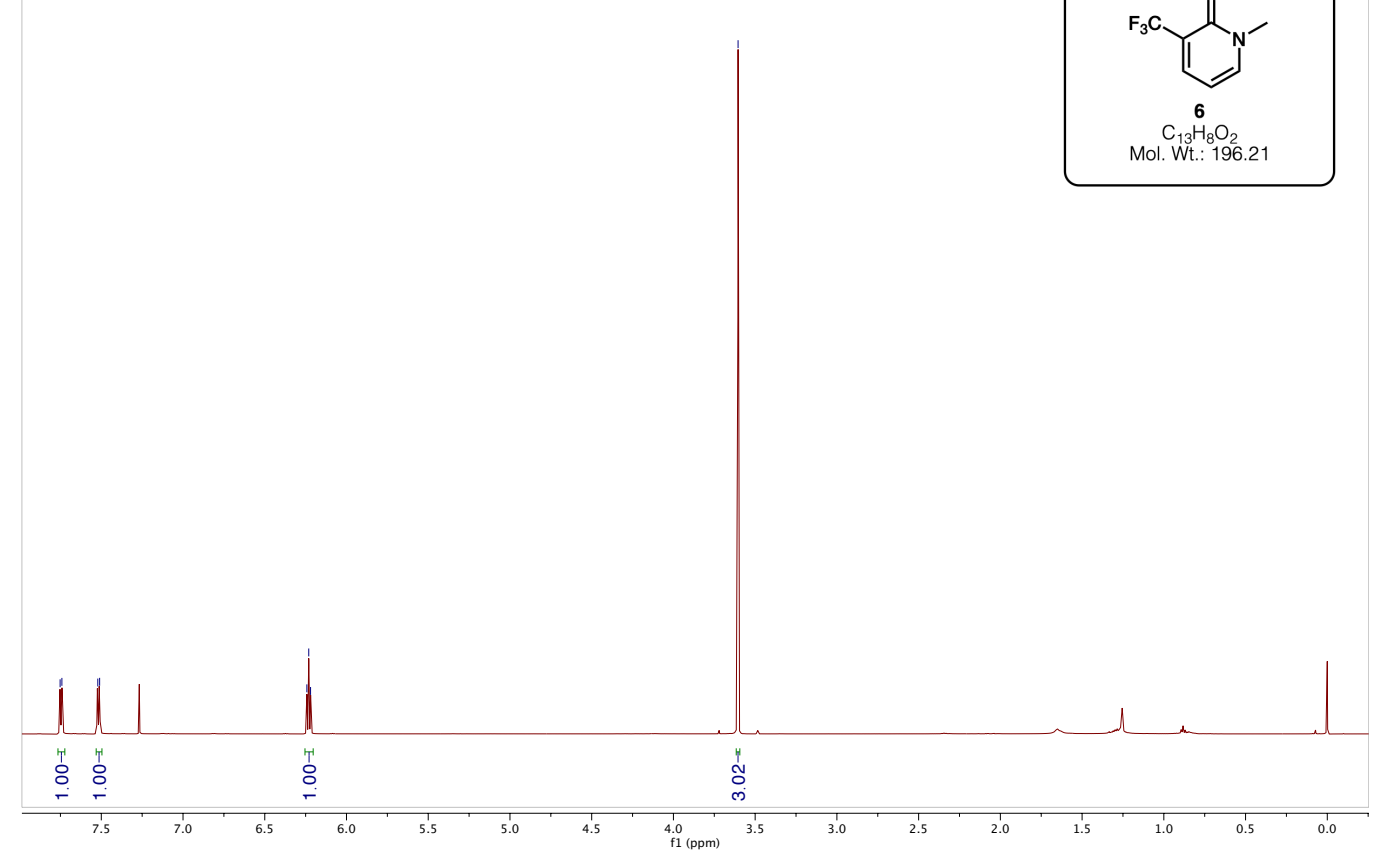

${ }^{19} \mathrm{~F}$ NMR (564 MHz, $\mathrm{CDCl}_{3}$ )

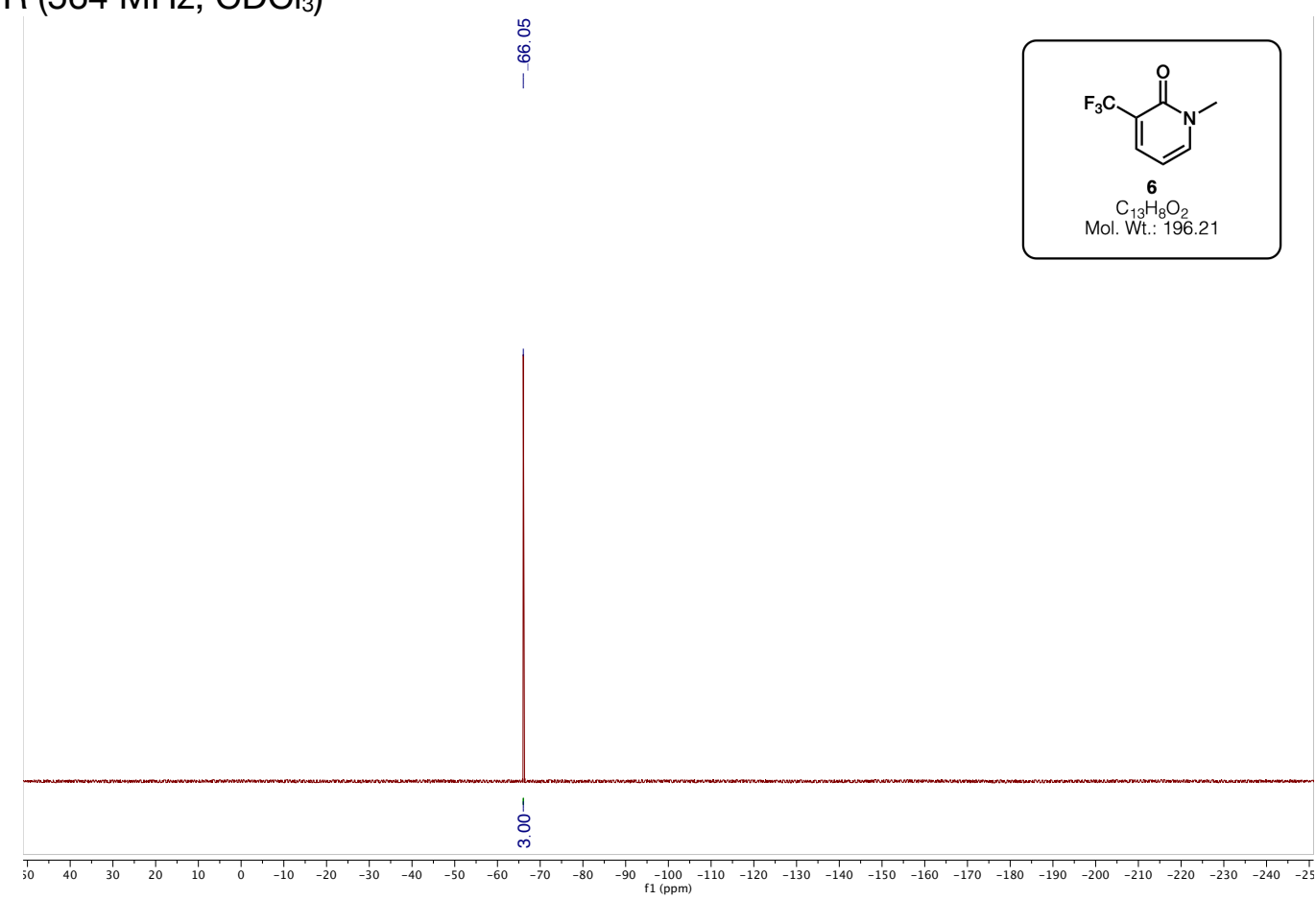


1,3,5-Trimethoxy-2-(trifluoromethyl)-benzene (8)

${ }^{1} \mathrm{H}$ NMR $\left(600 \mathrm{MHz}, \mathrm{CDCl}_{3}\right)$

$\stackrel{m}{i}$
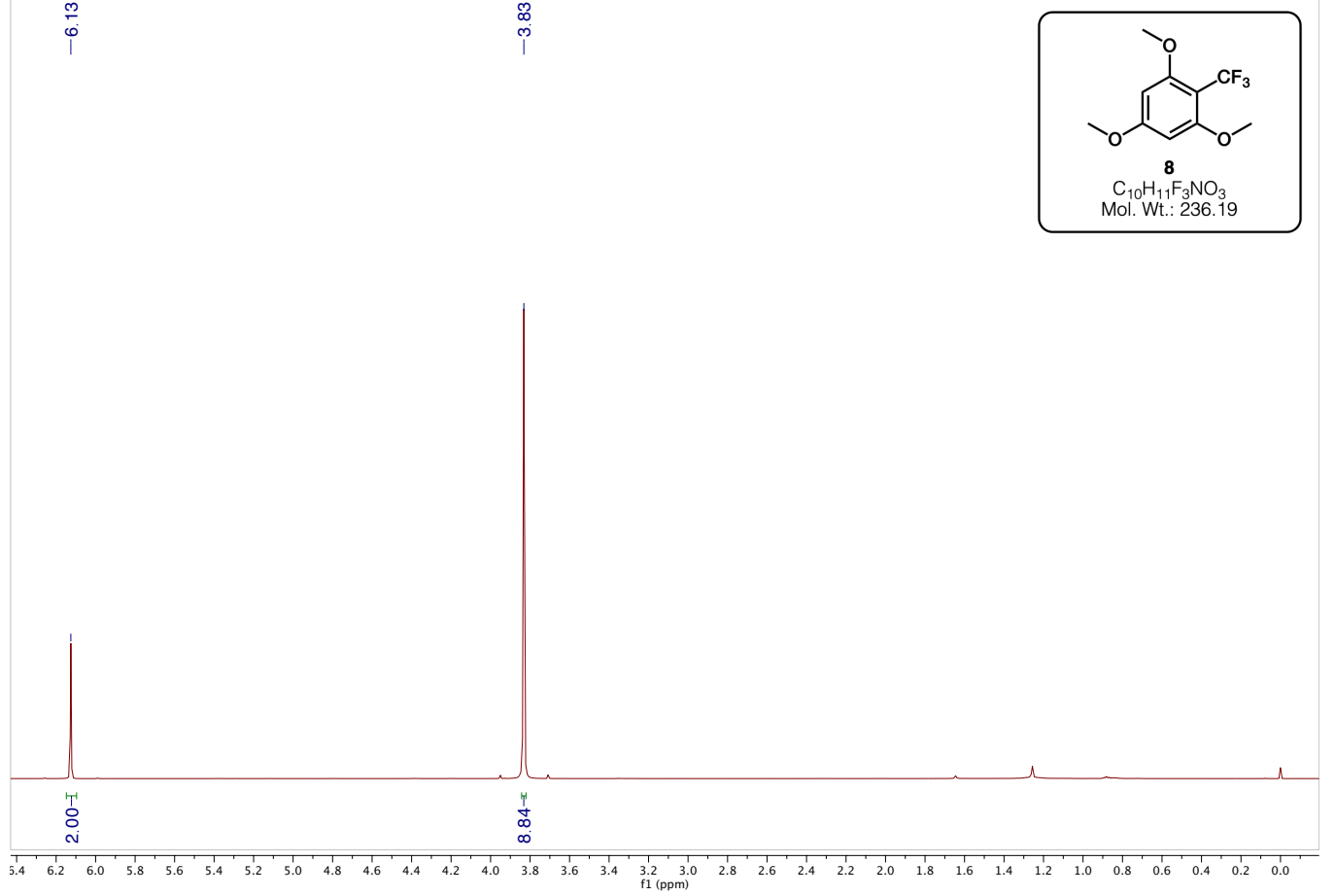

${ }^{13} \mathrm{C}$ NMR (151 MHz, $\left.\mathrm{CDCl}_{3}\right)$

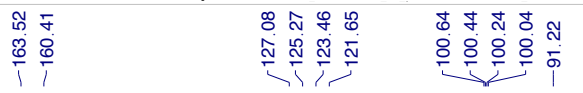

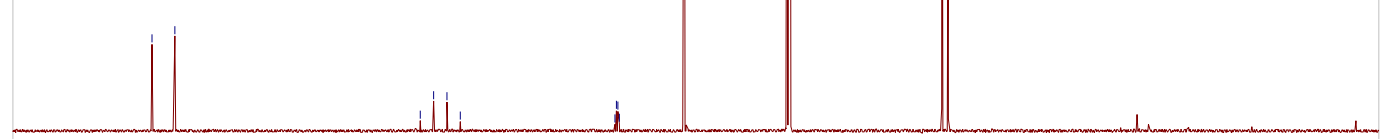

$$
\frac{1}{180}
$$

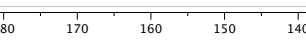


${ }^{19} \mathrm{~F} \mathrm{NMR}\left(376 \mathrm{MHz}, \mathrm{CDCl}_{3}\right)$

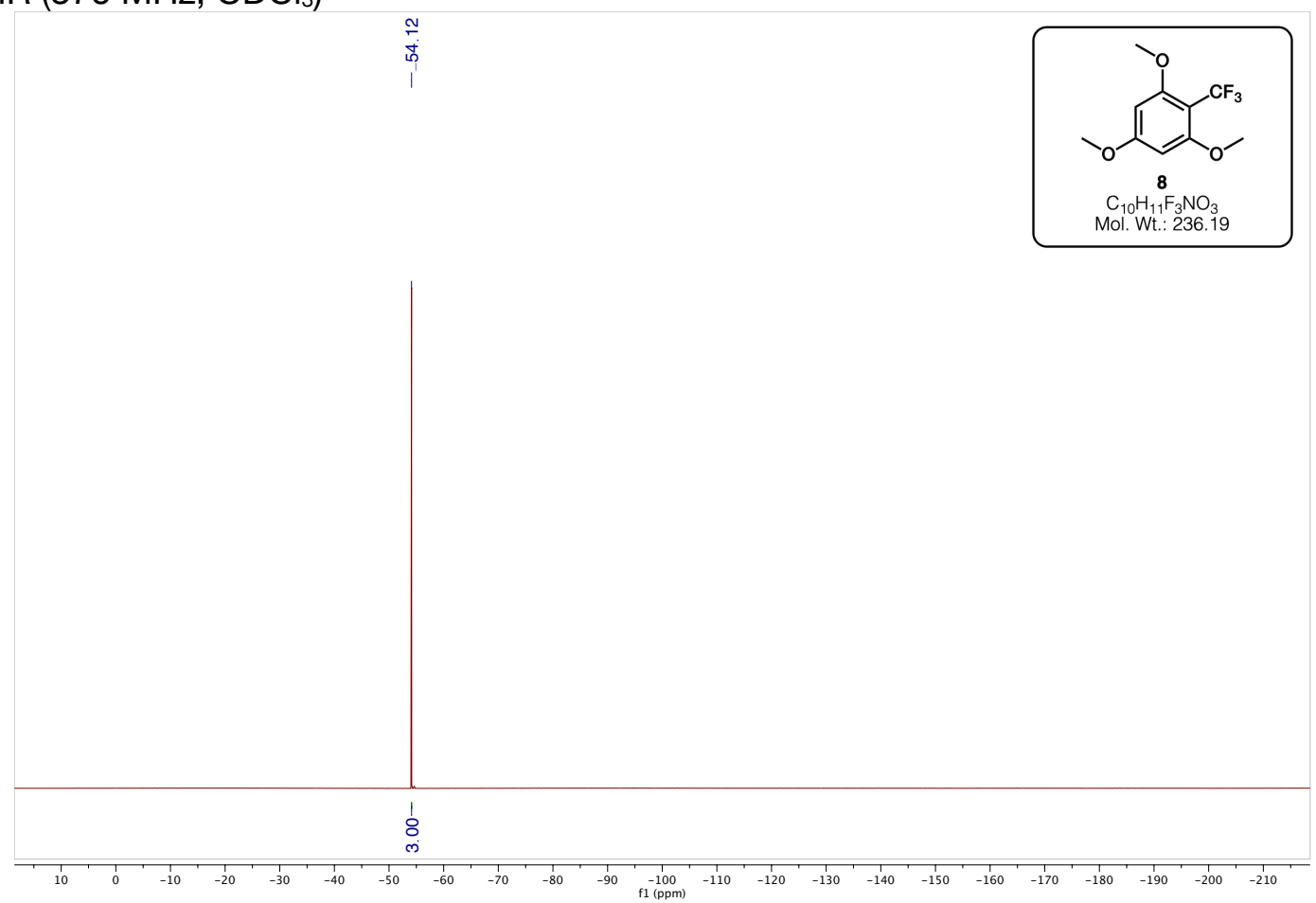

1-(tert-butyl) 2-methyl 5-(trifluoromethyl)-1H-pyrrole-1,2-dicarboxylate (10)

${ }^{1} \mathrm{H} \mathrm{NMR}\left(600 \mathrm{MHz}, \mathrm{CDCl}_{3}\right)$

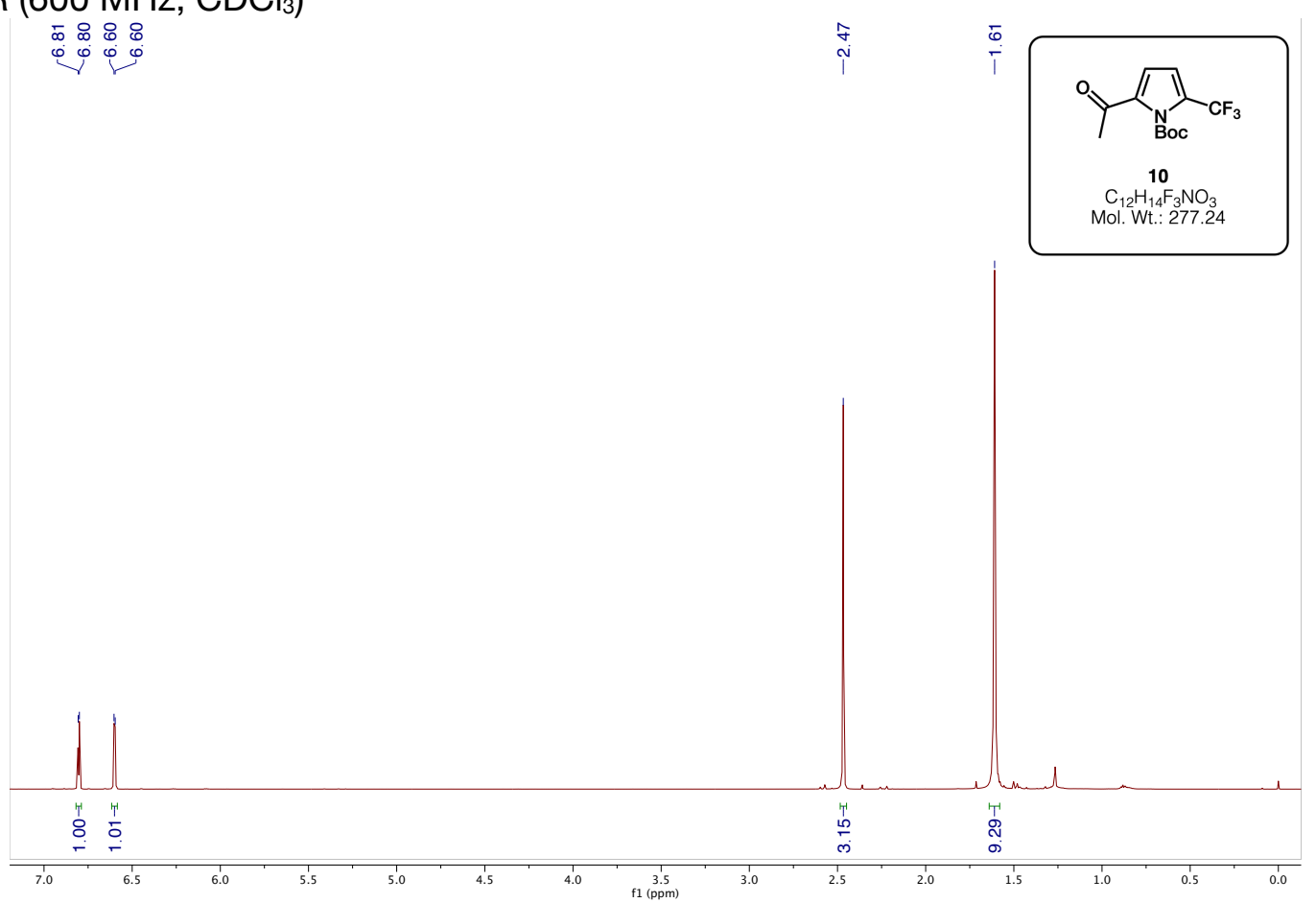


${ }^{13} \mathrm{C}$ NMR (151 MHz, $\left.\mathrm{CDCl}_{3}\right)$

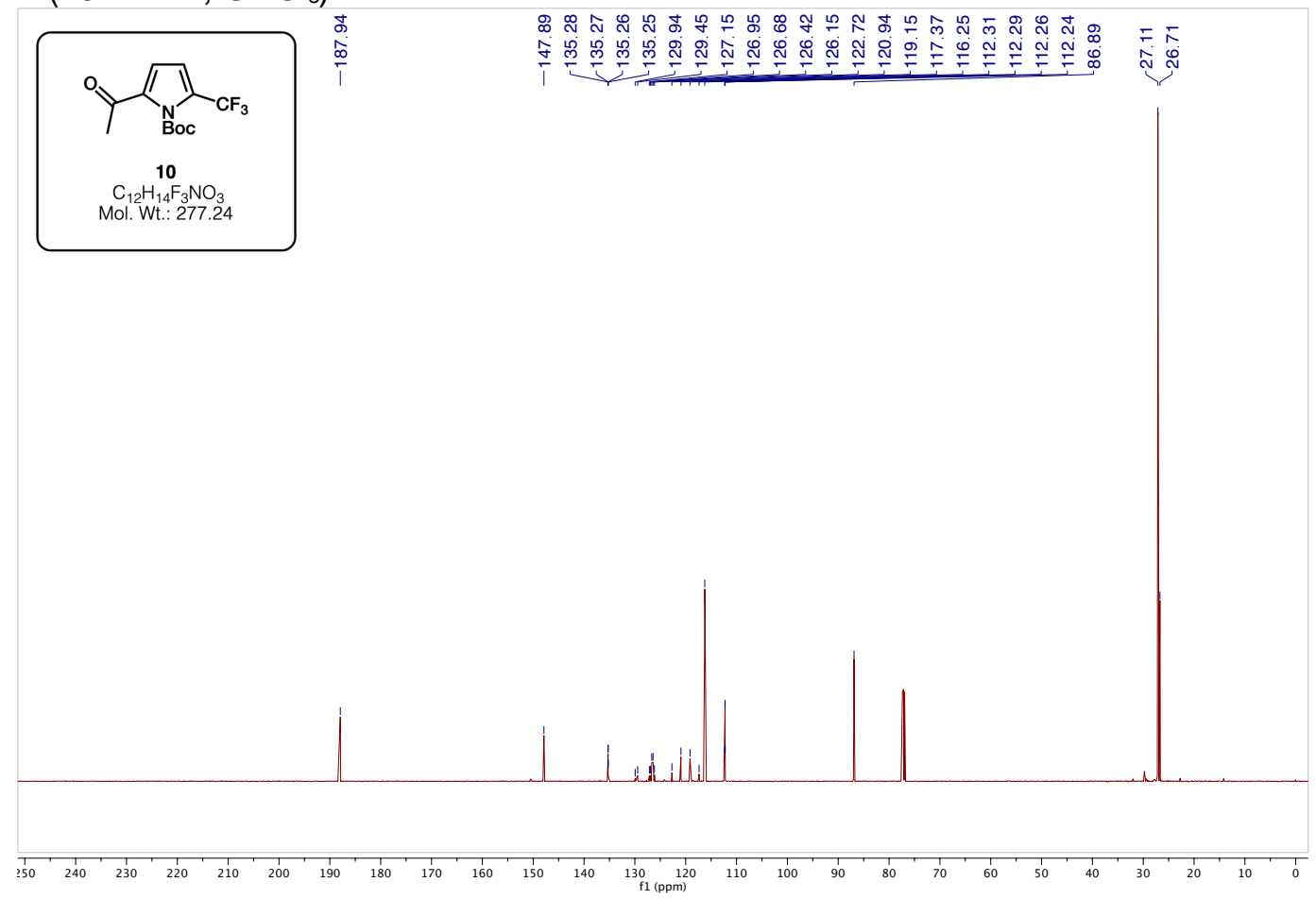

${ }^{19} \mathrm{~F}$ NMR (564 MHz, $\mathrm{CDCl}_{3}$ )

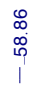
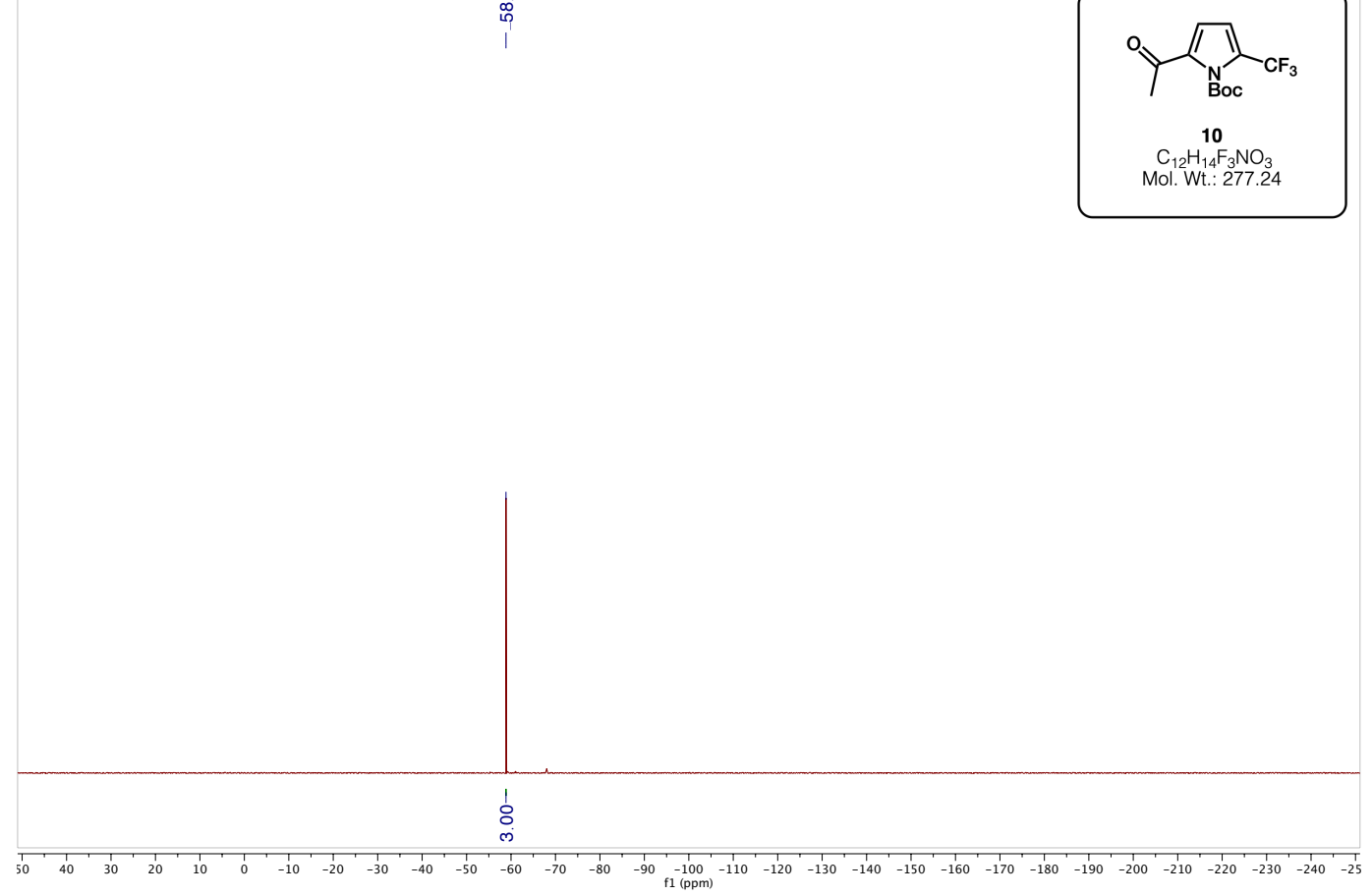


\section{References}

(1) Aung, T.; Liberko, C. A. Bringing Photochemistry to the Masses: A Simple, Effective, and Inexpensive Photoreactor, Right out of the Box. J. Chem. Educ. 2014, 91 (6), 939942.

(2) Douglas, J. J.; Albright, H.; Sevrin, M. J.; Cole, K. P.; Stephenson, C. R. J. A Visible-LightMediated Radical Smiles Rearrangement and Its Application to the Synthesis of a Difluoro-Substituted Spirocyclic ORL-1 Antagonist. Angew. Chemie - Int. Ed. 2015, 54 (49), 14898-14902.

(3) Li, L.; Mu, X.; Liu, W.; Wang, Y.; Mi, Z.; Li, C. J. Simple and Clean Photoinduced Aromatic Trifluoromethylation Reaction. J. Am. Chem. Soc. 2016, 138 (18), 5809-5812.

(4) Le, C. C.; Wismer, M. K.; Shi, Z. C.; Zhang, R.; Con-way, D. V.; Li, G.; Vachal, P.; Davies, I. W.; MacMillan, D. W. C. A General Small-Scale Reactor to Enable Standardization and Acceleration of Photocatalytic Reactions. ACS Cent. Sci. 2017, 3 (6), 647-653.

(5) Lim, C. H.; Kudisch, M.; Liu, B.; Miyake, G. M. C-N Cross-Coupling via Photoexcitation of Nickel-Amine Complexes. J. Am. Chem. Soc. 2018, 140 (24), 7667-7673.

(6) Morack, T.; Metternich, J. B.; Gilmour, R. Vitamin Ca-talysis: Direct, Photocatalytic Synthesis of Benzo-coumarins via (-)-Riboflavin-Mediated Electron Transfer. Org. Lett. 2018, 20 (5), 1316-1319.

(7) Ravetz, B. D.; Tay, N. E. S.; Joe, C. L.; Sezen-Edmonds, M.; Schmidt, M. A.; Tan, Y.; Janey, J. M.; Eastgate, M. D.; Rovis, T. Development of a Platform for Near-Infrared Photoredox Catalysis. ACS Cent. Sci. 2020, 6 (11), 2053-2059.

(8) Beatty, J. W.; Douglas, J. J.; Miller, R.; McAtee, R. C.; Cole, K. P.; Stephenson, C. R. J. Photochemical Perfluoroalkylation with Pyridine N-Oxides: Mechanistic Insights and Performance on a Kilogram Scale. Chem 2016, 1 (3), 456-472.

(9) Son, S.; Fu, G. C. Copper-Catalyzed Asymmetric [4+1] Cycloadditions of Enones with Diazo Compounds to Form Dihydrofurans. J. Am. Chem. Soc. 2007, 129 (5), 1046-1047

(10) Corcoran, E. B.; Pirnot, M. T.; Lin, S.; Dreher, S. D.; Dirocco, D. A.; Davies, I. W.; Buchwald, S. L.; Macmillan, D. W. C. Aryl Amination Using Ligand-Free Ni(II) Salts and Photoredox Catalysis. Science 2016, 353 (6296), 279-283. 


\section{Acknowledgments}

We thank Dr. Ilia A. Guzei for photography assistance. We are grateful to Sebastian Thompson for help fabricating custom LED stars. 